\title{
常温常压电催化合成氨的研究进展
}

\author{
詹溯章福祥* \\ (中国科学院大连化学物理研究所 大连 116023)
}

\begin{abstract}
摘要 氨不仅是重要的化肥化工原料, 还是理想的清洁能源载体. 目前人工氨合成主要基于 Haber-Bosch 过程, 但该方 法存在能耗大、转化率低、大量排放温室气体等问题. 相比而言, 利用太阳能催化转化 $\mathrm{N}_{2}$ 和 $\mathrm{H}_{2} \mathrm{O}$ 等制 $\mathrm{NH}_{3}$ 是一条实 现太阳能至化学能转化的绿色制氢储氢一体化路线, 受到世界各国科学家的高度关注. 但当前该技术路线的氮还原 (NRR)转化率和法拉第效率均较低, 开发高效 NRR 电催化剂并将其与捕光材料耦合是实现高效太阳能催化合成氨的 关键. 本综述将首先介绍太阳能催化合成氨的一些基本原理、主要技术路线和基本检测方法, 然后分类介绍传统热催 化剂、过渡金属催化剂、贵金属催化剂和非贵金属催化剂等在电催化 NRR 领域中的应用，以及提升 NRR 性能的主要 策略和其它氮源(如: $\mathrm{NO}_{3}{ }^{-}$和 $\mathrm{NO}$ )电催化合成氨的研究进展, 最后就该方向存在的一些问题以及急需突破的方向进行 了总结与展望.
\end{abstract}

关键词 合成氨; 电催化; 氮还原; 太阳能转化; 硝酸根还原; 一氧化氮还原

\section{Recent Progress on Electrocatalytic Synthesis of Ammonia Under Amibent Conditions}

\author{
Su Zhan Fuxiang Zhang* \\ (Dalian Institute of Chemical Physics, Chinese Academy of Sciences, Dalian 116023, China)
}

\begin{abstract}
Ammonia is not only an important chemical for fertilizer and industrial chemical, but also an ideal carrier of clean energy. Current ammonia synthesis is mainly based on Haber-Bosch process that suffers from some problems such as high energy consumption, low conversion and large amount of greenhouse gas emission, so the solar ammonia synthesis from $\mathrm{N}_{2}$ and $\mathrm{H}_{2} \mathrm{O}$ has recently attracted extensive attention. However, both conversion and Faradaic efficiency via the solar-based catalysis have been still retarded by poor surface catalysis process. Accordingly, development of efficient electrocatalysts for $\mathrm{N}_{2}$ reduciton reaction (NRR) as well as their coupling with photoadsorbers is highly desirable. The recent research progress in the following areas is summarized in this review: i) main electrocatalysts for NRR, including thermal catalyst, transition metal catalyst, noble metal catalyst and non-noble metal catalyst, ii) typical strategies to improve the NRR performance and iii) other routes using different nitrogen sources such as $\mathrm{NO}_{3}{ }^{-}$and NO. Finally, the remaining challenges and perspectives will be outlined.
\end{abstract}

Keywords ammonia synthesis; electrocatalysis; reduction of nitrogen; solar energy conversion; $\mathrm{NO}_{3}{ }^{-}$reduction; $\mathrm{NO}$ reduction

\section{1 引言}

合成氨对人类社会发展具有重要意义 ${ }^{[1]}$. 氨不仅是 重要的化肥和化工原料, 还是重要的清洁能源(氢)载 体 ${ }^{[2]}$. 氨的储氢容量为 $17.6 \%$ (质量分数), 能量密度可达 $3000 \mathrm{Wh} / \mathrm{kg}$, 是理想的储氢材料. 此外, $\mathrm{NH}_{3}$ 作为能源载 体还具有以下优势：首先, $\mathrm{NH}_{3}$ 易于运输和储存, 适用 于移动氢源, 在室温下压力为 $0.8 \mathrm{MPa}$ 左右时, $\mathrm{NH}_{3}$ 可以 液态形式储存; 其次, $\mathrm{NH}_{3}$ 分解产物为 $\mathrm{H}_{2}$ 与 $\mathrm{N}_{2}$, 不会对 环境造成其它污染 ${ }^{[3]}$; 最后, $\mathrm{NH}_{3}$ 相对安全, 不易爆炸. 目前, 世界每年的合成氨总量接近 2 亿吨, 随着人们对 环境和能源问题的重视, 对氨的需求还将逐年上升.
人工氨合成的主要方式是 Haber-Bosch 法 $^{[4]}$, 在高 温(650 750 K) 和高压 $(10 \mathrm{MPa})$ 环境中, 将 $\mathrm{N}_{2}$ 与 $\mathrm{H}_{2}$ 在催 化剂表面转化为 $\mathrm{NH}_{3}$. 该反应能耗高、反应条件苛刻, 单程转化率只有 $15 \%$ 左右, 据统计世界每年消耗 $2 \%$ 的 能量用于工业合成氨, 产生约 4 亿吨的 $\mathrm{CO}_{2}{ }^{[5]}$. 随着能 源和环境问题日益严峻，开发新型合成氨技术，降低反 应条件和能耗, 成为合成氨研究的发展趋势, 对人类社 会的可持续发展有重要影响.

为了降低合成氨能量消耗, 研究者们对常温常压 NRR 进行了大量探索研究 ${ }^{[6]}$. 太阳能是地球上最主要的 能量来源, 可满足氨合成的能量需求, 利用太阳能将 $\mathrm{N}_{2}$ 和 $\mathrm{H}_{2} \mathrm{O}$ 催化转化 $\mathrm{NH}_{3}$ 被认为是一条绿色的合成氨路线,

\footnotetext{
*E-mail: fxzhang@dicp.ac.cn

Received September 7, 2020; published November 30, 2020

Project supported by the National Natural Science Foundation of China (Nos. 21633009, 21925206, 21902159).

项目受国家自然科学基金(Nos. 21633009, 21925206, 21902159)资助.
} 
具有极大的开发潜力. 利用太阳能催化合成氨主要有三 条技术路线: 粉末光催化、光电催化、光伏发电与电催 化耦合等, 其不仅可实现太阳能至化学能的转化储存, 还可避免 $\mathrm{CO}_{2}$ 排放. 以光催化路线为例, 其太阳能转化 储存化学能的计算公式为:

$$
\begin{aligned}
& \eta=\left(\Delta G^{\theta} \times R\right) /\left(P_{\text {sun }} \times S\right) \\
& \mathrm{N}_{2}+3 \mathrm{H}_{2} \mathrm{O}=2 \mathrm{NH}_{3}+3 / 2 \mathrm{O}_{2} \quad \Delta G^{\theta}=678.8 \mathrm{~kJ} / \mathrm{mol}
\end{aligned}
$$

其中, $\eta$ 为太阳能至化学能转化效率; $\Delta G^{\theta}$ 为吉布斯自由 能变化; $R$ 为储存化学产物的生产速率; $P_{\text {sun }}$ 为太阳光辐 射强度; $S$ 为光照面积. 只有当 $\Delta G^{\theta}$ 远大于 0 的反应才能 实现太阳能至化学能的有效转化 ${ }^{[7]}$. 以水为质子源的合 成氨反应(式 2)在标准条件下的吉布斯自由能变化为 $678.87 \mathrm{~kJ} / \mathrm{mol}^{[8]}$, 可有效实现太阳能至化学能的转化. 图 1 是光催化反应合成氨示意图, 与光催化水分解制氢 相似, 光催化合成氨的基本过程分为捕光、电荷分离和 表面催化. 光能转化效率由材料的捕光效率、电荷分离 效率和表面催化效率乘积共同决定.

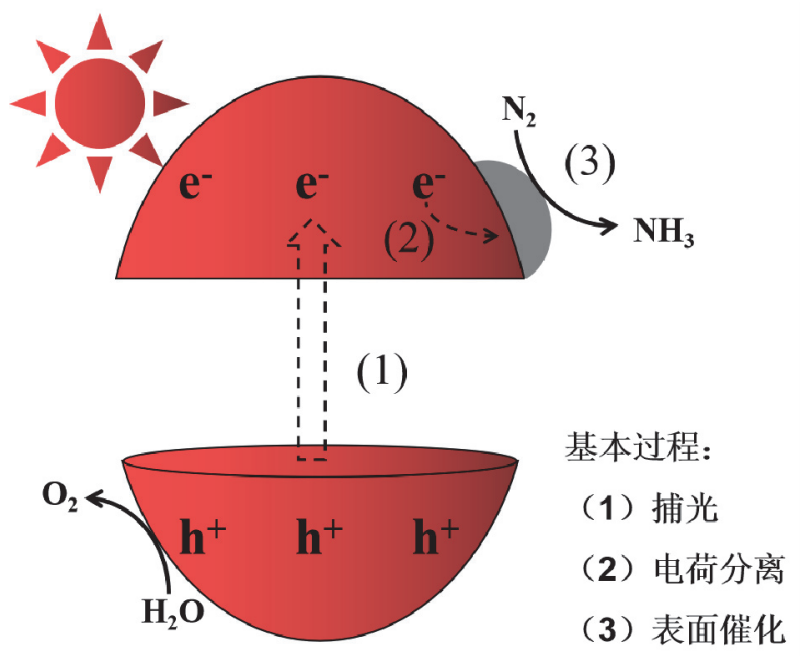

图 1 光催化反应合成氨示意图

Figure 1 Schematic diagram of photocatalytic ammonia synthesis

光催化合成氨面临的最主要的问题是缺少具有高 法拉第效率的 NRR 助催化剂, 大力发展 NRR 电催化剂 作为合成氨助剂是解决这一问题的重要手段. 迄今为 止，有多种材料被报道具有电催化 NRR 性能，但整体而 言电催化 NRR 产率和法拉第效率偏低, 合成氨助剂的 开发仍面临巨大挑战, 具体体现在: (1) $\mathrm{N}_{2}$ 作为稳定的

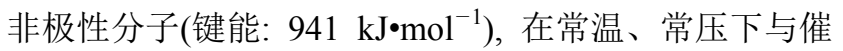
化剂相互作用弱, 不利于其化学吸附 ${ }^{[9]}$. (2) 由于质子参 与 $\mathrm{N}_{2}$ 活化, 析氢反应(HER)与 $\mathrm{NRR}$ 存在竞争关系 ${ }^{[10]}$, 进 一步限制了电催化合成氨效率. 开发高效稳定的 NRR 电催化剂是现阶段的研究重点. 针对如何开发 NRR 电 催化剂, 实现太阳能至化学能有效转化这一课题, 本文 概述了电催化 NRR 的研究现状, 重点介绍了 4 类有前景
的 NRR 催化剂的研究进展, 包括：传统热催化剂、固氮 酶同组分过渡金属催化剂、贵金属催化剂以及非贵金属 催化剂; 总结了几种提升 NRR 性能的策略; 并关注其 它不同氮源还原至 $\mathrm{NH}_{3}$ 的研究进展; 最后总结并展望了 太阳能催化 NRR 所面临的挑战及今后研究的趋势, 以 期为光催化 NRR 领域的研究工作提供一些启发.

\section{NRR 主要反应机制及产物检测}

\section{$2.1 N R R$ 主要反应机制}

催化剂表面进行的合成氨反应过程，可按照解离机 制和非解离机制进行. 图 2(a)是解离机制示意图 ${ }^{[11]}, \mathrm{N}_{2}$ 在催化剂表面首先发生解离生成活化 $* \mathrm{~N}, * \mathrm{~N}$ 逐步加氢 最终生成 $\mathrm{NH}_{3}$. 由于 $\mathrm{N} \equiv \mathrm{N}$ 键中第一个 $\mathrm{N}-\mathrm{N}$ 键的解离 能高达 $410 \mathrm{~kJ} \bullet \mathrm{mol}^{-1}, \mathrm{~N}_{2}$ 解离活化必须要在高温环境下 进行, 是 Haber-Bosch 过程的主要反应机制, $\mathrm{N}_{2}$ 的活化 被认为是该过程的决速步 ${ }^{[12]}$.

\section{A Dissociative Pathway}

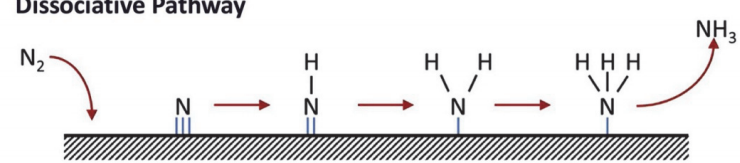

B Associative Pathway

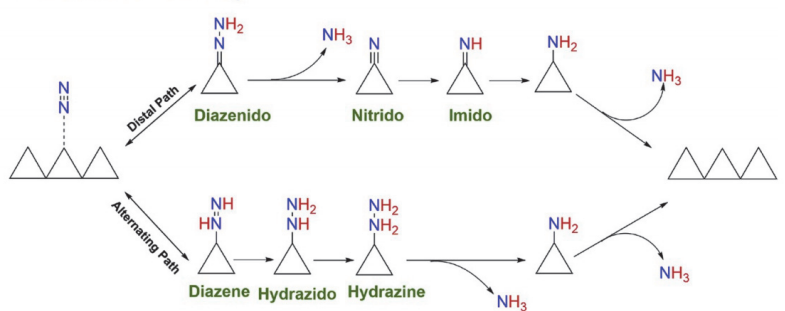

图 2 NRR 反应机理和路径: (A)解离活化; (B)非解离活化

Figure 2 Reaction and mechanisms pathways proposed for NRR: (A) Dissociative pathway; (B) associative pathway

温和条件进行的 $N R R$ 不足以使 $\mathrm{N} \equiv \mathrm{N}$ 键直接断裂, NRR 主要以非解离机制进行. 图 2(b)是非解离机制示意 图 ${ }^{[13]}, \mathrm{N}_{2}$ 分子吸附在催化剂表面后首先进行加氢反应, 随后发生 $\mathrm{N} \equiv \mathrm{N}$ 键断裂, 最终生成 $\mathrm{NH}_{3}$; 在液相反应中, 质子将参与 $\mathrm{N}_{2}$ 活化, 降低反应势垒. 表 1 总结了 $\mathrm{NRR}$ 加氢反应对应的还原电势 ${ }^{[14]}$, 从中可以看出由 $\mathrm{N}_{2}$ 到

表 1 NRR 加氢反应对应的还原电势

Table 1 Hydrogenation reaction for NRR with the corresponding reduction potentials

\begin{tabular}{ll}
\hline 反应式 & 电位/V (vs. NHE), $\mathrm{pH}=0$ \\
\hline $\mathrm{N}_{2}+\mathrm{e}^{-} \rightarrow \mathrm{N}_{2}^{-}$ & -4.16 \\
$\mathrm{~N}_{2}+\mathrm{H}^{+}+\mathrm{e}^{-} \rightarrow \mathrm{N}_{2} \mathrm{H}$ & -3.20 \\
$\mathrm{~N}_{2}+2 \mathrm{H}^{+}+2 \mathrm{e}^{-} \rightarrow \mathrm{N}_{2} \mathrm{H}_{2}$ & -1.10 \\
$\mathrm{~N}_{2}+4 \mathrm{H}^{+}+4 \mathrm{e}^{-} \rightarrow \mathrm{N}_{2} \mathrm{H}_{4}$ & -0.36 \\
$\mathrm{~N}_{2}+5 \mathrm{H}^{+}+5 \mathrm{e}^{-} \rightarrow \mathrm{N}_{2} \mathrm{H}_{5}^{+}$ & -0.23 \\
$\mathrm{~N}_{2}+8 \mathrm{H}^{+}+6 \mathrm{e}^{-} \rightarrow 2 \mathrm{NH}_{4}^{+}$ & 0.27 \\
$\mathrm{~N}_{2}+6 \mathrm{H}^{+}+6 \mathrm{e}^{-} \rightarrow \mathrm{NH}_{3}$ & 0.55 \\
\hline
\end{tabular}


$\mathrm{NH}_{3}$ 至少需要 6 个 $\mathrm{e}^{-}$和 6 个 $\mathrm{H}^{+}$参与反应, 而 $\mathrm{H}^{+}$还原无 论从热力学还是动力学都比 $\mathrm{N}_{2}$ 还原容易, 因此 HER 与 $\mathrm{NRR}$ 为竞争反应, 在这一过程中 $\mathrm{N}_{2}$ 的活化、中间产物 加氢速率均可能成为反应决速步. 非解离机制是生物固 氮、光/电催化合成氨的主要方式.

\subsection{NRR 产物检测}

NRR 产物检测常用方法有: 显色法(包括纳氏试剂 分光光度法 ${ }^{[15]}$ 和水杨酸-次氯酸分光光度法 $)^{[16]}$, 离子色 谱 ${ }^{[17]},{ }^{15} \mathrm{~N}$ 同位素标记-核磁共振 NMR 等 ${ }^{[18]}$. 表 2 总结了 常用的几种 NRR 产物检测方法的特点与注意事项.

纳氏试剂分光光度法是利用碘离子和录离子在强 碱性条件下与 $\mathrm{NH}_{4}{ }^{+}$发生配位反应, 其如反应式(3)所示, 配合物在 $420 \mathrm{~nm}$ 处具有特征吸收峰, 通过比较吸收峰 强度可用于测定 $\mathrm{NH}_{4}{ }^{+}$浓度, $\mathrm{NH}_{4}{ }^{+}$检测范围为 $0.025 \sim 5.0$ $\mathrm{mg} / \mathrm{L}^{[19]}$.

$$
2 \mathrm{~K}_{2} \mathrm{HgI}_{4}+\mathrm{NH}_{3}+3 \mathrm{KOH} \rightarrow \mathrm{Hg}_{2} \mathrm{NH}_{2} \mathrm{I}+7 \mathrm{KI}+2 \mathrm{H}_{2} \mathrm{O}
$$

水杨酸-次氯酸分光光度法是在碱性介质中, $\mathrm{NH}_{4}{ }^{+}$ 依次与水杨酸盐, 次氯酸根离子和亚硝基铁氰化钠(硝 普纳)发生反应生成蓝色化合物, 其特征吸收峰在 664 $\mathrm{nm}$ 处，该方法检测 $\mathrm{NH}_{4}{ }_{4}^{+}$范围为 $0.01 \sim 1.0 \mathrm{mg} / \mathrm{L}^{[19]}$.

离子色谱也广泛被应用于 $\mathrm{NH}_{4}{ }^{+}$检测, 其检测范围 为 $0.02 \sim 40 \mathrm{mg} / \mathrm{L}$, 此方法具有重现性好、灵敏度高、检 测时间短等优点, 但使用离子色谱测定 $\mathrm{NH}_{4}{ }^{+}$含量时, 需要注意 $\mathrm{NH}_{4}{ }^{+}$与 $\mathrm{Na}^{+}$离子出峰位置接近, 导致分离度 低, 如电解液中 $\mathrm{Na}^{+}$离子含量过高, 将无法准确测定 $\mathrm{NH}_{4}{ }^{+}$浓度 ${ }^{[20]}$.

为得到相对可靠的结果，除显色法和离子色谱外， 还需要使用 ${ }^{15} \mathrm{~N}$ 同位素-核磁共振 NMR 对实验结果进行 验证. 通过同位素标定可直接对生成氨的来源进行追 踪, 以确定催化剂活性, 其检测范围为 $5 \sim 10 \mu \mathrm{mol} / \mathrm{L}^{[21]}$. ${ }^{15} \mathrm{~N}$ 同位素-核磁共振 NMR 测定的结果应与显色法或离 子色谱的结果近似, 通过多种检测结果相互验证, 确定 催化剂合成氨产率.

由于在常温常压条件下合成氨产率较低,一些含氮 催化剂或杂质氮源将对实验结果产生严重干扰, 因此必 须要对催化剂进行稳定性和重复性测试. 对于稳定性测 试, 催化剂持续合成氨时间应 $>5 \mathrm{~h}$, 且单位活性中心上 转化了的反应物分子数(TON) 应大于 1 , 并至少重复 3 次 ${ }^{[22]}$. 此外需在实验过程中加以注意的杂质氮源有:
（1）反应气. 一些高纯氮气中可能含有 $\mathrm{NO}_{x}$ 污染物, $\mathrm{NO}_{x}$ 比 $\mathrm{N}_{2}$ 更容易还原生成氨, 因此需要用合适的吸收 剂去除 $\mathrm{NO}_{x}$. (2) Nafion 膜 ${ }^{[23]}$. 电催化 NRR 实验常用的 Nafion 膜也可能干扰实验结果, 实验过程中累积的 $\mathrm{NH}_{4}{ }^{+}$可能与酸发生离子交换而释放出 $\mathrm{NH}_{4}{ }^{+}$. (3) 空气 中的氨. 氨是常用的化学药剂, 因此实验室空气中含有 的氨 $\left(0.05 \sim 250 \mathrm{mg} / \mathrm{L}\right.$ 量级 $\left.{ }^{[24]}\right)$ 可能在电解液中累积, 从 而影响 NRR 结果. (4) 人呼吸中含有 $0.3 \sim 3.0 \mathrm{mg} / \mathrm{L}$ 量级 的氨 ${ }^{[25]}$.

为避免杂质对实验结果的干扰, 得到相对准确的结 果, Andersen 等 ${ }^{[26]}$ 制定了严格的测试标准流程, 如图 3 所示.

\section{NRR 电催化剂研究进展}

\section{1 传统热催化剂在电催化 NRR 中应用}

$\mathrm{Fe} 、 \mathrm{Ru}$ 基催化剂是常用的商用氨合成催化剂，也是 被研究的最为广泛的合成氨催化剂[27]. 对于活性组分、 载体结构、助剂种类等的研究, 可为电催化剂开发设计 提供宝贵经验. 随着近年来光电催化合成氨研究的兴 起, $\mathrm{Fe} 、 \mathrm{Ru}$ 基催化剂在常温、常压合成氨领域也表现出 巨大的应用前景.

对于热催化反应而言, $\mathrm{Fe}$ 基催化剂活性中心是由 7 个相邻的 $\mathrm{Fe}$ 原子组成 $(\mathrm{C} 7 \text { 位 })^{[28]}$, 活性仅与 $\mathrm{C} 7^{[29]}$ 数量有 关, 且 $\mathrm{Fe}$ 基催化剂只有在高压条件下才能表现出良好 的活性 ${ }^{[30]}$. 这是由于 $\mathrm{N}_{2}$ 在 $\mathrm{Fe}$ 表面解离活化后, 形成的 $\mathrm{Fe}-\mathrm{N}$ 结构相对稳定, 在低压条件下不利于 $* \mathrm{~N}$ 加氢脱附. 因此直接将 $\mathrm{Fe}$ 基热催化剂用于电催化 $\mathrm{NRR}$, 催化剂表 现出的活性极低. 例如 $5 \sim 10 \mathrm{~nm}$ 的 $\mathrm{Fe}_{2} \mathrm{O}_{3} /$ /碳纳米管 $\left(\mathrm{Fe}_{2} \mathrm{O}_{3}-\mathrm{CNT}\right)$ 催化剂在 $-2.0 \mathrm{~V}$ vs. $\mathrm{Ag} / \mathrm{AgCl}$ 电位下合成 氨产率仅为 $2.18 \times 10^{-3} \mathrm{~g}_{\mathrm{NH} 3} \cdot \mathrm{m}^{-2} \cdot \mathrm{h}^{-1}$, 法拉第效率不足 $0.025 \%$, 明显低于其他 NRR 电催化剂性能 ${ }^{[31]}$. 图 4 是 $\mathrm{Fe}_{2} \mathrm{O}_{3}-\mathrm{CNT}$ 电催化 NRR 装置示意图. $\mathrm{Fe}_{2} \mathrm{O}_{3}-\mathrm{CNT}$ 装载在 聚合电解质膜(PEM) 型电解池进行反应, 电解池两侧以 Nafion 膜-气体扩散电极(GDL) 隔开, 阳极一侧使用 2 $\mathrm{mol} / \mathrm{L} \mathrm{KOH}$ 为电解液, 反应式: $2 \mathrm{H}_{2} \mathrm{O} \rightarrow \mathrm{O}_{2}+4 \mathrm{H}^{+}+4 \mathrm{e}^{-}$; 阴极一侧充入高纯 $\mathrm{N}_{2}$, 反应式: $\mathrm{N}_{2}+6 \mathrm{H}^{+}+6 \mathrm{e}^{-} \rightarrow 2 \mathrm{NH}_{3}$. 这种电解池的优势在于 Nafion 膜可隔绝 $\mathrm{H}_{2} \mathrm{O}$ 与催化剂 直接接触，并将阳极一侧的 $\mathrm{H}^{+}$传递到阴极催化剂表面 参与 $\mathrm{N}_{2}$ 的活化，因此在一定程度上能抑制析氢反应. 尽

表 2 NRR 产物检测方法对比

Table 2 A comparison of NRR product determination methods

\begin{tabular}{|c|c|c|c|}
\hline 检测方法 & 检测范围 & 特点 & 注意事项 \\
\hline $\begin{array}{l}\text { 纳氏试剂显色 } \\
\text { 法 }\end{array}$ & $0.025 \sim 5.0 \mathrm{mg} / \mathrm{L}$ & 操作简便 & $\begin{array}{l}\text { 试剂中含采, 毒性高, 对环境危害大, 试剂寿命短, 需要注意 } \mathrm{Fe}^{2+} 、 \\
\mathrm{Cu}^{2+} 、 \mathrm{Ca}^{2+} \text { 等离子对显色效果干扰 }\end{array}$ \\
\hline $\begin{array}{l}\text { 水杨酸-次氯酸 } \\
\text { 显色法 }\end{array}$ & $0.01 \sim 1.0 \mathrm{mg} / \mathrm{L}$ & 操作简便、低毒性 & $\begin{array}{l}\text { 溶液 } \mathrm{pH} \text { 应大于 } 11 \text {, 否则将影响显色化合物生成, 需要注意 } \mathrm{Fe}^{2+} \text { 离子 } \\
\text { 对显色效果干扰 }\end{array}$ \\
\hline 离子色谱 & $0.02 \sim 40 \mathrm{mg} / \mathrm{L}$ & $\begin{array}{l}\text { 重现性好、灵敏度高、检测 } \\
\text { 时间短 }\end{array}$ & 注意电解液中 $\mathrm{Na}^{+}$浓度, 减少对 $\mathrm{NH}_{4}{ }^{+}$检测的干扰 \\
\hline $\begin{array}{l}{ }^{15} \mathrm{~N} \text { 同位素-核磁 } \\
\text { 共振 NMR }\end{array}$ & $5 \sim 10 \mu \mathrm{mol} / \mathrm{L}$ & $\begin{array}{l}\text { 为生成氨的真实来源提供 } \\
\text { 直接证据 }\end{array}$ & 需保证 ${ }^{15} \mathrm{~N}_{2}$ 气体的纯度 \\
\hline
\end{tabular}




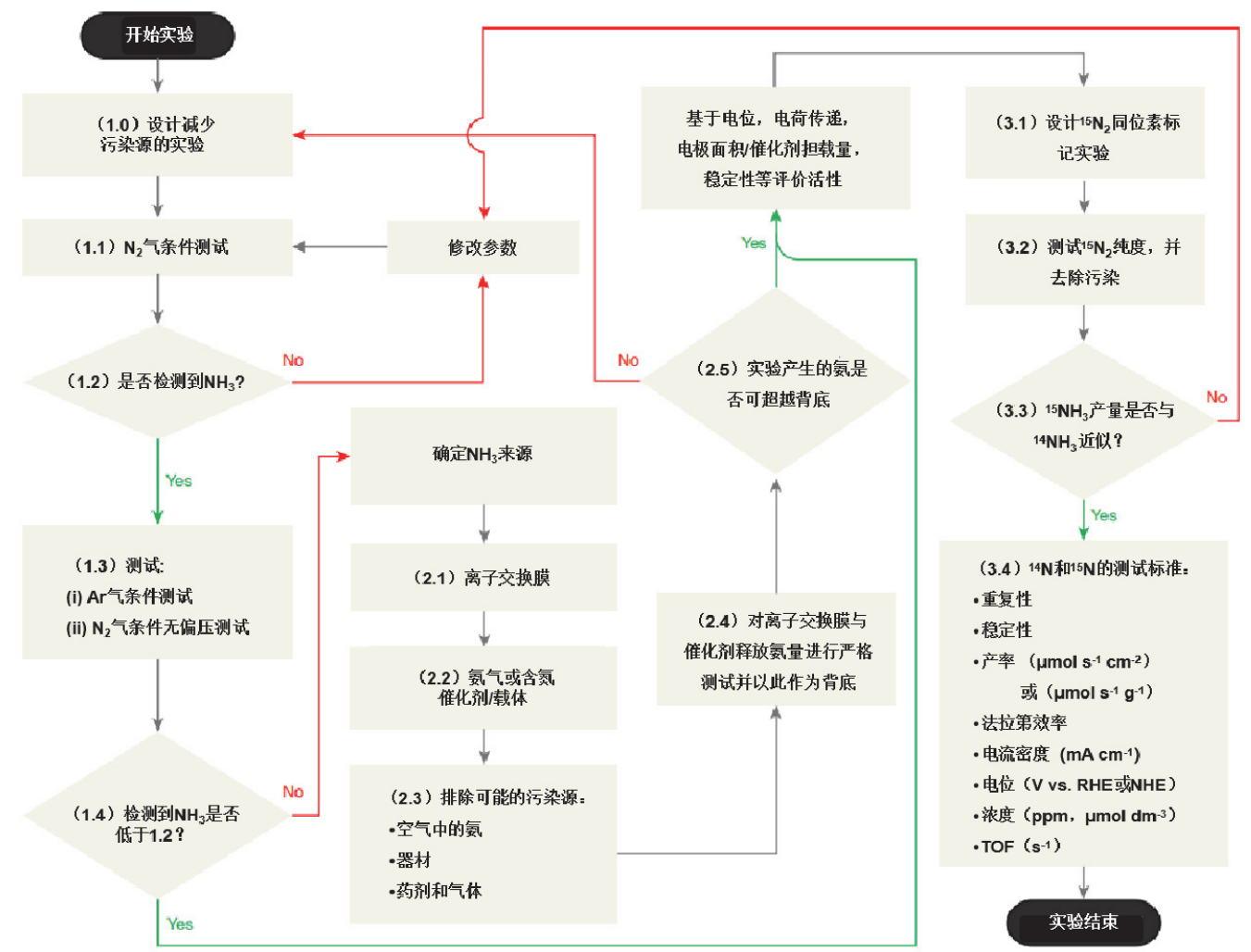

图 3 电催化合成氨实验标准流程

Figure 3 Suggested protocol for the benchmarking of electrochemical nitrogen reduction

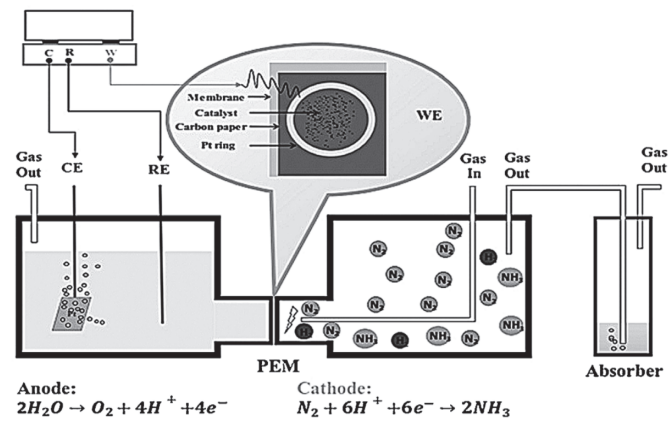

图 4 电催化合成氨流动反应器示意图, 阴极在无电解液(气相)环境 中运行, PEM 为质子交换膜

Figure 4 Shematic view of the electrocatalytic flow reactor for ammonia synthesis, with the cathode cell operating under electrolyte-less conditions (gas phase). PEM indicates proton exchange membrane

管如此, 催化剂在合成氨选择性方面依旧很低, 这项工 作表明 $\mathrm{Fe}$ 基热催化剂无法在常温常压下有效实现氨合 成. 而近年来随着单原子催化的发展 ${ }^{[32]}$, 使 $\mathrm{Fe}$ 基催化 剂在常温常压下有效进行 NRR 成为了可能. Zhang 等 ${ }^{[33]}$ 发现当 $\mathrm{Fe}$ 的尺寸下降到原子级别时, $\mathrm{Fe}$ 基催化剂可表 现出较高的 NRR 性能, 具有单原子结构的 $\mathrm{Fe}-\left(\mathrm{O}-\mathrm{C}_{2}\right)_{4}$ 催化剂合成氨活性可达 $32.1 \mu \mathrm{g} \cdot \mathrm{h}^{-1} \cdot \mathrm{mg}_{\mathrm{cat}}{ }^{-1}$ (5350 $\mu \mathrm{g}$ • $\mathrm{h}^{-1} \cdot \mathrm{mg}_{\mathrm{Fe}}{ }^{-1}$ ), 法拉第效率为 $29.3 \%$, 其活性远远高于具 有纳米尺度的 $\mathrm{Fe}$ 基催化剂, 经过长达 $40 \mathrm{~h}$ 的稳定性测 试和 10 次的重复性测试, 催化剂能保持良好活性. 除对 活性中心进行调控外, 选择适宜的载体将对催化产物选
择性有显著影响. Wang 等 ${ }^{[34]}$ 设计了具有 $\mathrm{Fe}-\mathrm{N}_{4}-\mathrm{C}$ 结构的 单原子催化剂, 将单原子 $\mathrm{Fe}$ 基催化剂合成氨法拉第效 率提高至 $56.55 \%$. 通过 DFT 计算对具有单原子结构的 $\mathrm{Fe}$ 基催化剂 NRR 过程进行了解释, $\mathrm{N}_{2}$ 在催化剂表面吸 附后, $\mathrm{N}_{2}$ 分子与 $\mathrm{Fe}$ 原子间距约为 $0.45 \mathrm{~nm}$, 这一距离可 保证 $\mathrm{N}_{2}$ 在催化剂表面有效活化. 此外在 $\mathrm{Fe}-\mathrm{N}_{4}-\mathrm{C}$ 表面质 子还原的势垒高达 $2.91 \mathrm{eV}$, 说明 $\mathrm{Fe}$ 单原子表面不利于 HER 进行, 因此单原子结构的 $\mathrm{Fe}$ 基催化剂可在常温常 压下表现出较高的电催化合成氨性能.

相比于 $\mathrm{Fe}$ 基催化剂, $\mathrm{Ru}$ 基催化剂可在低压条件下 实现氨的合成, 被誉为第二代合成氨热催化剂, 是合成 氨研究领域的明星材料 ${ }^{[35]}$. 对于热催化反应, $\mathrm{Ru}$ 基催化 剂活性中心由 5 个相邻的 $\mathrm{Ru}$ 原子构成(B5 位) ${ }^{[36]}$, 催化 剂活性只与 $\mathrm{B} 5^{[37]}$ 位数量有关, 但高昂成本是制约其应 用的最主要原因. 近年来, 有研究表明具有单原子结构 的 $R u$ 基催化剂在常温常压 NRR 研究中表现出巨大的潜 力. Peng 等 ${ }^{[38]}$ 以 $2 \mathrm{D}$ MXene 为载体担载 $\mathrm{Ru}$ 单原子 ( $\mathrm{Ru}-\mathrm{Mo}_{2} \mathrm{CT}_{x}$ ), 基于扩展 $\mathrm{X}$ 射线精细结构吸收(EXAFS) 谱结果分析, 单原子 $\mathrm{Ru}$ 可能与 $\mathrm{C}$ 或 $\mathrm{O}$ 形成配位. $\mathrm{Ru}-\mathrm{Mo}_{2} \mathrm{CT}_{x}$ 在电位为 $-0.3 \mathrm{~V}$ vs. RHE 时, 合成氨速率为 $40.57 \mu \mathrm{g} \cdot \mathrm{h}^{-1} \cdot \mathrm{mg}^{-1}$, 法拉第效率为 $25.77 \%$. DFT 计算显 示具有单原子结构的 $\mathrm{Ru}$ 可促进电子由 $\mathrm{Ru} 3 \mathrm{~d}$ 轨道向吸 附 $\mathrm{N} 2 \mathrm{p}$ 轨道转移, 降低反应势垒. 对于单原子催化剂而 言，除单原子中心本身外，配位环境也可显著影响 NRR 
性能. Geng 等 ${ }^{[39]}$ 将含 Ru 的 MOF (ZIF-8)热解后, 得到具 有 $\mathrm{Ru}-\mathrm{N}$ 结构的单原子催化剂 $(\mathrm{Ru} \mathrm{SAs} / \mathrm{N}-\mathrm{C})$, 以 0.05 $\mathrm{mol} / \mathrm{L} \mathrm{H}_{2} \mathrm{SO}_{4}$ 为电解液, 在 $-0.2 \mathrm{~V}$ vs. RHE 电位下, 具 有 $\mathrm{Ru}-\mathrm{N}$ 结构的催化剂合成氨法拉第效率为 $29.6 \%$, 产 率高达 $120.9 \mu \mathrm{g}_{\mathrm{NH} 3} \cdot \mathrm{mg}_{\text {cat. }}{ }^{-1} \cdot \mathrm{h}^{-1}$, 且连续测试 $12 \mathrm{~h}$ 后, 催 化剂表现出良好的稳定性, 明显优于 Ru-C 结构的催化 剂.

无论在热催化还是电催化过程, $\mathrm{Fe} 、 \mathrm{Ru}$ 是有效的 $\mathrm{N}_{2}$ 活化催化剂. 现有研究显示, 具有单原子结构的 Fe、 $\mathrm{Ru}$ 基催化剂更适用于常温常压合成氨领域. 单原子结 构保证 $\mathrm{N}_{2}$ 有效活化的同时, 还可在一定程度上抑制 HER, 因此表现出较高的合成氨产率和法拉第效率. 但 整体来说, $\mathrm{Fe} 、 \mathrm{Ru}$ 催化剂的设计开发仍有极大的提升空 间, 借鉴热催化剂的研究, 单原子 $\mathrm{Fe} 、 \mathrm{Ru}$ 基催化剂在优 化载体与选取助剂方面需开展大量探索工作.

\section{2 过渡金属钼、钒基催化剂}

原核生物可利用自身的固氮酶将空气中的 $\mathrm{N}_{2}$ 还原 至 $\mathrm{NH}_{3}$, 该过程具有反应条件温和、 $\mathrm{N}_{2}$ 转化效率高等特 点. 钼铁固氮酶 ${ }^{[40]}$ 和钒铁氮酶 ${ }^{[41]}$ 是两种最为普遍的固 氮酶体系, 钼、钒作为生物固氮酶中活性位点的组成元 素对开发常温常压人工固氮材料有重要借鉴意义. 研究 者针对钼基, 钒基催化剂展开了研究, 验证了其可在常 温常压条件下实现 NRR.

Zhao 等 ${ }^{[42]}$ 以 Mo 原子嵌入氮化嗍 $(\mathrm{BN})$ 的 B 空位处 (Mo-BN) 为模型分析了其 NRR 过程, 结果如图 5 所示. 单原子 Mo 的优势体现在: $\mathrm{N}_{2}$ 在单原子 $\mathrm{Mo}$ 表面的活化 过电位仅需 $0.19 \mathrm{~V}$, 可有效活化 $\mathrm{N}_{2}$; 另一方面对加氢过 程中形成的中间产物 $\mathrm{N}_{2} \mathrm{H}^{*} 、 \mathrm{NH}_{2}$ *进行分析, 单原子 $\mathrm{Mo}$ 对 $\mathrm{N}_{2} \mathrm{H}$ *有较高的吸附能, 而对 $\mathrm{NH}_{2}$ *吸附能较低, 这有 利于活化 $\mathrm{N}^{*}$ 在其表面加氢脱附. 理论计算表明 $\mathrm{N}_{2}$ 在单 原子 Mo 表面的反应机制与固氮酶机理类似, 有可能实 现高效 NRR. Han 等 ${ }^{[43]}$ 以钼酸铵和盐酸差胺为原料, 在 $\mathrm{Ar}$ 气保护中进行热解, 将 Mo 以单原子形式分散在 $\mathrm{N}$ 掺 杂的多孔碳(SA-Mo/NPC)中. 通过 X 射线吸收近边结构 (XANES)谱、X 射线光电子能谱(XPS)和 EXAFS 结果分 析, 单原子 $\mathrm{Mo}$ 主要与 $\mathrm{C}$ 和 $\mathrm{N}$ 成键, 生成 Mo-C 和 Mo-N 结构, $\mathrm{Mo}$ 的价态为 $82.5 \%$ 的 $\mathrm{Mo}^{6+}$ 和 $17.5 \%$ 的 $\mathrm{Mo}^{4+}$. 以 $0.1 \mathrm{~mol} / \mathrm{L} \mathrm{KOH}$ 为电解液, 在一 $0.3 \mathrm{~V}$ vs. RHE 电位下, $\mathrm{SA}-\mathrm{Mo} / \mathrm{NPC}$ 合成氨速率为 $(34.0 \pm 3.6) \mu \mathrm{g} \bullet \mathrm{h}^{-1} \cdot \mathrm{g}_{\text {cat. }}{ }^{-1}$, 法 拉第效率为 $14.6 \% \pm 1.6 \%$, 经过 $50000 \mathrm{~s}$ 稳定性测试后, 合成氨产率及法拉第效率未出现明显衰减, 验证了单原

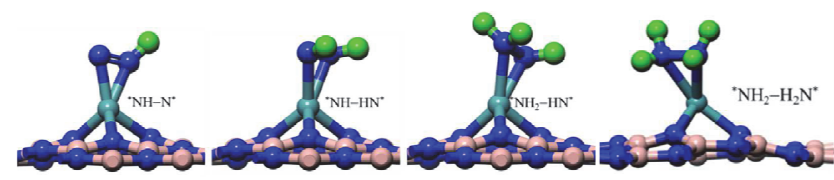

图 5 Mo-BN 表面 NRR 过程

Figure 5 NRR proceeded on Mo-BN monolayer
子 Mo 结构可实现常温常压 NRR. 进一步的理论研究表 明, 精准调控 Mo 单原子的配位环境可能是提升 NRR 性 能的关键. Huang 等 ${ }^{[44]}$ 以具有单原子结构的 Mo-BN 作为 $\mathrm{N}_{2}$ 活性中心, 将其嵌入石墨烯表面 $(\mathrm{Mo} @ \mathrm{BCN})$, 改变 Mo-BN 结构的配位环境. DFT 计算显示, 随着 B-C 和 $\mathrm{N}-\mathrm{C}$ 键数量增多, $\mathrm{Mo} @ \mathrm{BCN}$ 对 $\mathrm{N}_{2}$ 的吸附能从 -0.22 $\mathrm{eV}$ 提升至 $-0.65 \mathrm{eV}$, 而对 $\mathrm{H}$ 的吸附能维持在 $-0.32 \mathrm{eV}$, 通过改变 Mo-BN 的配位环境提升了的 NRR 选择性. 如 何在材料制备过程中精准调控 Mo 的配位环境, 是实现 Mo 基催化剂高效 NRR 的关键.

钒固氮酶是自然界中第二种生物固氮酶, 一些研究 者针对钒基催化剂在常温常压下的 NRR 性能展开了研 究. Feng 等 ${ }^{[45]}$ 的研究表明具有低价态的钒基催化剂可实 现常温常压 $\mathrm{NRR}$. 以 $\mathrm{NH}_{4} \mathrm{VO}_{3}$ 为钒源、 $\mathrm{C}_{6} \mathrm{H}_{12} \mathrm{O}_{6}$ 为碳源, 以 $\mathrm{Ar}$ 为保护气, 在 $1000{ }^{\circ} \mathrm{C}$ 下进行退火, 得到碳化钒 $\left(\mathrm{V}_{8} \mathrm{C}_{7}\right)$ 催化剂. XPS 结果显示, $\mathrm{V}$ 以为 0 价和 +4 价混合 形式稳定存在, 在 $0.1 \mathrm{~mol} / \mathrm{L} \mathrm{HCl}$ 电解液中, $-0.4 \mathrm{~V}$ vs. $\mathrm{RHE}$ 电位下, $\mathrm{V}_{8} \mathrm{C}_{7}$ 合成氨产率为 $34.62 \mu \mathrm{g} \cdot \mathrm{h}^{-1} \cdot \mathrm{cm}^{-2}$, 法 拉第效率为 $12.20 \%$. 此外, $\mathrm{Qu}$ 等 ${ }^{[46]}$ 在 $\mathrm{NH}_{3}$ 气氛中对 $\mathrm{V}_{2} \mathrm{O}_{5}$ 进行氮化处理, 得到氮化钒 $(\mathrm{VN})$ 纳米线催化剂. 在 $0.1 \mathrm{~mol} / \mathrm{L} \mathrm{HCl}$ 溶液中, 电位为 $-0.3 \mathrm{~V}$ vs. RHE, 测得催 化剂 NRR 产率为 $2.48 \times 10^{-10} \mathrm{~mol}^{-1} \cdot \mathrm{s}^{-1} \cdot \mathrm{cm}^{-2}$, 法拉第效 率为 $3.58 \%$. 但需要注意的是, 对于 $\mathrm{VN}$ 催化剂的 $\mathrm{NRR}$ 性能还存在争议 ${ }^{[47]}, \mathrm{V}-\mathrm{N}$ 结构在电化学过程中可能被破 坏从而影响电化学结果, 因此要验证 $\mathrm{VN}$ 是否是有效的 $\mathrm{N}_{2}$ 活化催化剂还需要使用 ${ }^{15} \mathrm{~N}$ 进行确认. 对于钒基催化 剂的开发, 目前面临的最主要问题是如何使低价态的钒 在电催化过程中稳定存在.

整体而言, 研究生物固氮酶的组分、结构和原理, 并将这些原理与电催化技术耦合, 从而开发可在常温常 压下高效氨合成催化剂, 这一技术路线极具研究潜力且 充满挑战. 无论是钼基固氮酶还是钒基固氮酶对其机理 还缺乏充分认知, 对于相应的电催化剂开发需要进行大 量的实验工作.

\section{3 贵金属催化剂}

贵金属催化剂一直是催化领域研究的重点. 贵金属 未填满的 $\mathrm{d}$ 轨道具有很强的给电子能力, 使得贵金属催 化剂表现出较高的活性, 此外贵金属催化剂还具有抗氧 化、耐腐蚀等优点. 一些贵金属如 $\mathrm{Au}^{[23]} 、 \mathrm{Pd}^{[48]} 、 \mathrm{Ru}^{[39]}$ 等被证实具有电催化 NRR 性能, 其中 $\mathrm{Au}$ 被认为是一种 有前景的 NRR 电催化剂. 通常来讲, $\mathrm{Au}$ 是化学惰性的, 难以进行催化反应, 但当 $\mathrm{Au}$ 的尺寸减小到纳米级别时, $\mathrm{Au}$ 可表现出催化性能 ${ }^{[49]}$. Bao 等 ${ }^{[50]}$ 合成了暴露 $\{730\}$ 晶 面族的 $\mathrm{Au}$ 纳米棒, $\{730\}$ 晶面族由(210)和(310)晶面构成 (如图 6 所示). DFT 计算显示阶梯状 $\{730\}$ 晶面族可提供 $\mathrm{N}_{2}$ 活化位点, 在一 $0.2 \mathrm{~V}$ vs. RHE 电位下, $\mathrm{Au}$ 纳米棒合成 氨产率为 $1.648 \mu \mathrm{g} \cdot \mathrm{h}^{-1} \cdot \mathrm{cm}^{-2}$, 法拉第效率为 $4 \%$. 当 $\mathrm{Au}$ 
催化剂的尺寸进一步下降到原子级别时, $\mathrm{Au}$ 催化剂的 NRR 性能将明显提升. Wang 等 ${ }^{[51]}$ 的工作表明具有原子 级别分散的 $\mathrm{Au}$ 催化剂, 其 NRR 性能要比 $\mathrm{Au}$ 纳米颗粒 催化剂高 22.5 倍, 在一 $0.1 \mathrm{~V}$ vs. RHE 电位下, 产氨速率 为 $1305 \mu \mathrm{g} \cdot \mathrm{h}^{-1} \cdot \mathrm{mg}_{\mathrm{Au}}{ }^{-1}$, 法拉第效率为 $11.1 \%$.

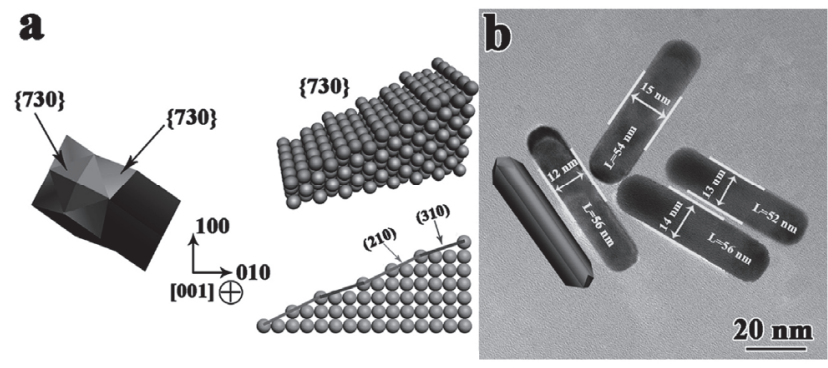

图 $6 \mathrm{Au}$ 纳米棒原子结构示意图. (a) 暴露 $\{730\}$ 晶面族的 $\mathrm{Au}$ 纳米棒 几何模型; (b) 金纳米棒的 TEM 图

Figure 6 Atomic level surface structures of Au THH NR. (a) Geometric models of an Au THH NR and exposed $\{730\}$ facet; (b) TEM image of $\mathrm{Au}$ THH NRs

贵金属 Pt 也可实现电催化 NRR. 由于 $\mathrm{Pt}$ 具有优异 的 HER 性能 ${ }^{[52]}$, 会抑制 NRR 进行, 早期的研究认为 Pt 不适宜作为 NRR 催化剂. 而 Hao 等 ${ }^{[53]}$ 的研究表明具有 单原子结构的 Pt 也可用于 NRR. 将 Pt 以单原子担载在 $\mathrm{WO}_{3}$ 纳米片上, DFT 计算显示 Pt-3O 结构对* $\mathrm{H}$ 和 $\mathrm{N}_{2}$ 的 吸附能分别为 $-0.94 \mathrm{eV}$ 和 $-1.34 \mathrm{eV}$, 这表明具有单原 子结构的 $\mathrm{Pt}$ 基催化剂, NRR 选择性将得到提升. 在 0.1 $\mathrm{mol} / \mathrm{L} \mathrm{K}_{2} \mathrm{SO}_{4}$ 电解液中, 电位为一 $-0.2 \mathrm{~V}$ vs. RHE 时, 单 原子 $\mathrm{Pt}$ 合成氨产率为 $342.4 \mu \mathrm{g} \bullet \mathrm{h}^{-1} \bullet \mathrm{mg}_{\mathrm{Pt}}{ }^{-1}$, 法拉第效率 为 $31.3 \%$, 是 $\mathrm{Pt}$ 纳米催化剂的 15 倍(2.1\%). 图 7 是 $\mathrm{Pt}$ 单原子催化剂和 $\mathrm{Pt}$ 纳米催化剂在 $\mathrm{Ar}$ 气饱和的 $\mathrm{K}_{2} \mathrm{SO}_{4}$ 电 解液中的线性扫描伏安曲线(LSV), 该结果也证明了具 有单原子结构的 Pt 会降低 HER 选择性.

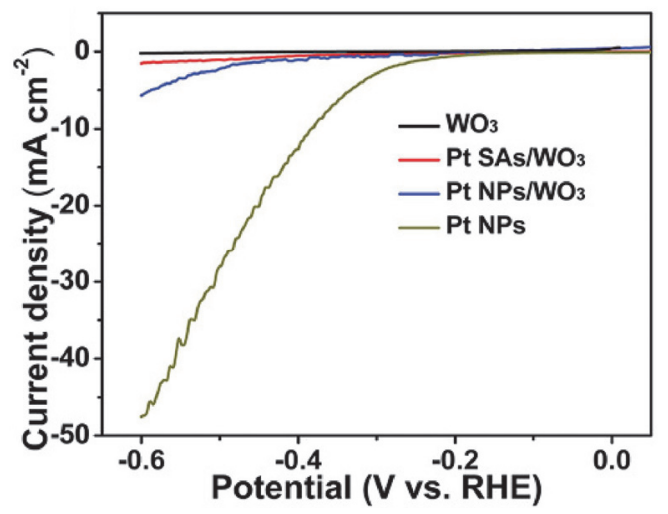

图 7 各样品在 $\mathrm{Ar}$ 气饱和的 $0.1 \mathrm{~mol} / \mathrm{L} \mathrm{K}_{2} \mathrm{SO}_{4}$ 电解液中的线性扫描伏 安曲线(LSV)

Figure 7 Linear sweep voltammetric (LSV) curves of each samples in Ar-saturated $0.1 \mathrm{~mol} / \mathrm{L} \mathrm{K}_{2} \mathrm{SO}_{4}$ electrolyte

综上所述, 作为催化领域研究的重点材料, 贵金属 催化剂在电催化 NRR 方向上也表现出一定的潜力. 单 原子贵金属催化剂无论在 $\mathrm{N}_{2}$ 活化效率, 还是产物选择
性方面都有明显改善，但整体而言仍有待提升，需要进 一步优化设计催化剂结构、提升贵金属分散程度.

\section{4 非贵金属催化剂}

虽然贵金属催化剂表现出一定的研究潜力，但高成 本一直是限制其应用的主要原因. 因此设计和开发非贵 金属催化剂是 NRR 领域研究的另一重点 ${ }^{[54]}$. Bi 基催化 剂是非贵金属催化剂研究中的热门材料. 研究显示具有 纳米片结构的 $\mathrm{Bi}$ 基催化剂, 层间 $\mathrm{Bi}-\mathrm{Bi}$ 间距被压缩, 可 有效促进 $\mathrm{Bi}$ 的 $\mathrm{p}$ 轨道电子离域, 因此可将 $\mathrm{N}_{2}$ 吸附和活 化 ${ }^{[55]}$. Li 等通过电化学法制备得到 $\mathrm{Bi}$ 纳米片 $(\mathrm{Bi} \mathrm{NS}$ )和 $\mathrm{Bi}$ 纳米颗粒 $(\mathrm{Bi} \mathrm{NP})$, 在 NRR 测试中, Bi NP 的合成氨速 率仅为 $\mathrm{Bi} N \mathrm{NS}$ 的 1/9 左右, 证明了 $\mathrm{Bi}$ 纳米片更有利于 NRR 过程. Zhang 等 ${ }^{[56]}$ 的工作也表明 $\mathrm{Bi}$ 纳米片具有较好 的 $\mathrm{NRR}$ 性能, 生长在 $\mathrm{Cu}$ 䇴上的 $\mathrm{Bi}$ 纳米片阵列 $(\mathrm{Bi}$

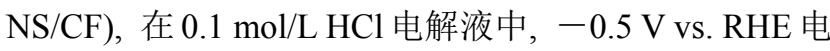
位下, $\mathrm{Bi} \mathrm{NS} / \mathrm{CF}$ 合成氨速率为 $6.89 \times 10^{-11} \mathrm{~mol}^{-1} \cdot \mathrm{s}^{-1}$. $\mathrm{cm}^{-2}$, 法拉第效率为 $10.26 \%$. 在经历过 6 次循环测试和 $24 \mathrm{~h}$ 稳定性测试后, Bi NS/CF 活性没有明显降低, 表明 催化剂具有良好的稳定性. 对 NRR 测试后的样品进行 XPS 分析, 催化剂含有 0 价 $\mathrm{Bi}$, 表明 $\mathrm{Bi}$ 单质是 $\mathrm{N}_{2}$ 催化 活化中心. 使用理论计算分析 $\mathrm{N}_{2}$ 在 $\mathrm{Bi}(012) 、(104)$ 和 (110)表面上的 NRR 过程自由能分布(图 8), 结果表明 NRR 过程主要发生在 Bi 的(012)表面. 此外, 一些非贵 金属虽然本身不具有 NRR 性能，但通过掺杂可对其他 材料电子结构进行调控，从而使催化剂表现出更好的 NRR 性能. 具有氧空位 $\left(V_{O}\right)$ 的 $\mathrm{TiO}_{2}$ 被报道具有 $\mathrm{NRR}$ 性 能 ${ }^{[57]}, \mathrm{Wu}$ 等 ${ }^{[54 \mathrm{a}]}$ 发现利用 +1 和 +2 价 $\mathrm{Cu}$ 可以调节 $\mathrm{V}_{\mathrm{O}}$ 浓度和 $\mathrm{Ti}^{3+}$ 缺陷态 $\left(\mathrm{Cu}-\mathrm{TiO}_{2}\right)$, 由于化学补偿效应, 可诱 导过渡金属氧化物中不同的缺陷态，显著提升 $\mathrm{TiO}_{2}$ $\mathrm{NRR}$ 性能. 相比于 $\mathrm{TiO}_{2}, \mathrm{Cu}-\mathrm{TiO}_{2}$ 在 $-0.55 \mathrm{~V}$ vs. RHE 电 位下, 合成氨产率从 $4.45 \mu \mathrm{g} \cdot \mathrm{h}^{-1} \cdot \mathrm{mg}_{\text {cat. }}{ }^{-1}$ 提升至 21.31 $\mu \mathrm{g} \cdot \mathrm{h}^{-1} \cdot \mathrm{mg}_{\text {cat. }}{ }^{-1}$ ，法拉第效率从 $2.96 \%$ 提升至 $21.99 \%$. 经 过 5 次循环实验, $\mathrm{Cu}-\mathrm{TiO}_{2}$ 活性未发生明显衰减. 非贵金 属催化剂由于其成本低, 储量丰富等优势一直是催化领 域研究的重点，但相比于贵金属催化剂，非贵金属给电 子能力较差, 不利于 $\mathrm{N}_{2}$ 活化, 通常需引入杂原子对过渡 金属 $\mathrm{d}$ 轨道态密度进行调控, 以增强过渡金属给电子能 力 $^{[58]}$. 因此在选用匹配的杂原子对非贵金属催化剂结 构进行精准调控方面仍需进行大量工作，此外易发生 HER 竞争也是非贵金属催化剂亟待解决的问题. 为便 于比较催化剂性能，各类催化剂的合成氨条件、产率、 法拉第效率总结于表 3 .

\section{4 提升 NRR 性能策略}

\section{1 低析氢选择性材料用于 NRR}

尽管 NRR 电催化剂开发已经取得长足发展，但依 旧面临 HER 竞争的问题. 液相 NRR 过程, 质子的还原 

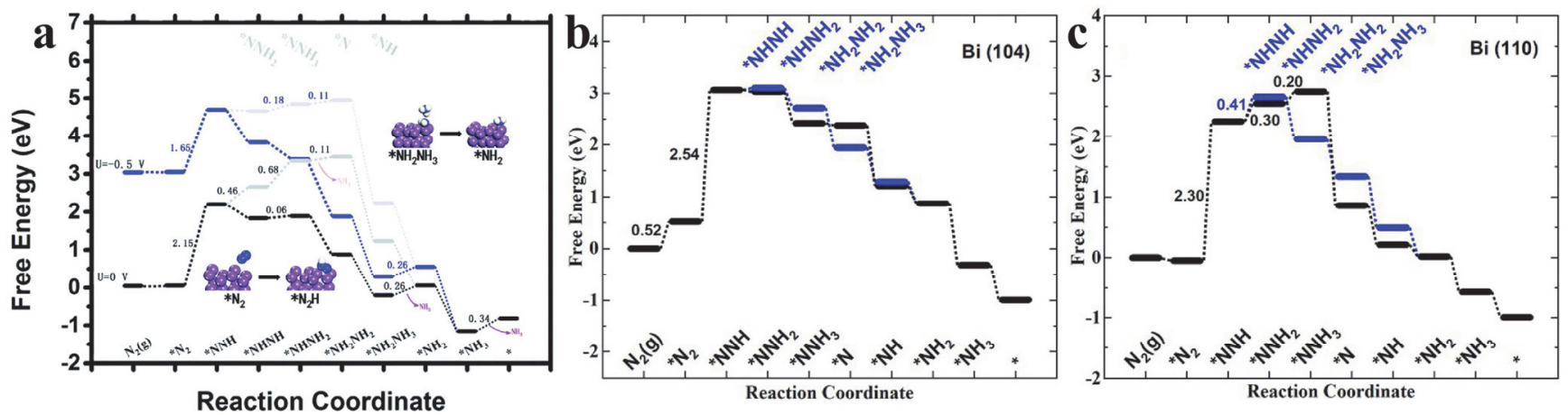

图 8 (a) $\mathrm{Bi}(012) 、$ (b) $\mathrm{Bi}(104)$ 和(c) $\mathrm{Bi}(110)$ 表面上的 NRR 过程自由能分布

Figure 8 Free energy profile of NRR process on (a) Bi (012), (b) Bi(104) and (c) Bi(110)

表 3 电催化剂性能对比

Table 3 Summary of NRR electrocatalysts

\begin{tabular}{|c|c|c|c|c|c|}
\hline & 催化剂 & 电解液 & $\mathrm{NH}_{3}$ 产率 & 法拉第效率/\% & 文献 \\
\hline \multirow[t]{6}{*}{ 传统热催化剂 } & $\mathrm{Fe}_{2} \mathrm{O}_{3} / \mathrm{CNT}$ & $\mathrm{KHCO}_{3}$ & $2.18 \times 10^{-3} \mathrm{~g}_{\mathrm{NH} 3} \cdot \mathrm{m}^{-2} \cdot \mathrm{h}^{-1}(-2.0 \mathrm{~V}$ vs. $\mathrm{Ag} / \mathrm{AgCl})$ & 0.025 & [31] \\
\hline & $\mathrm{Fe}-\left(\mathrm{O}-\mathrm{C}_{2}\right)_{4}$ & $0.1 \mathrm{~mol} / \mathrm{L} \mathrm{KOH}$ & $32.1 \mu \mathrm{g} \cdot \mathrm{h}^{-1} \cdot \mathrm{mg}_{\text {cat }}{ }^{-1}(-0.1 \mathrm{~V}$ vs. RHE $)$ & 29.3 & [33] \\
\hline & $\mathrm{Fe}-\mathrm{N}_{4}-\mathrm{C}$ & $0.1 \mathrm{~mol} / \mathrm{L} \mathrm{KOH}$ & $7.48 \mu \mathrm{g} \cdot \mathrm{h}^{-1} \cdot \mathrm{mg}^{-1}(0.0 \mathrm{~V}$ vs. RHE $)$ & 56.55 & [34] \\
\hline & $\mathrm{Fe}-\mathrm{W}_{18} \mathrm{O}_{49}$ & $0.25 \mathrm{~mol} / \mathrm{L} \mathrm{LiClO}_{4}$ & $24.7 \mu \mathrm{g} \cdot \mathrm{h}^{-1} \cdot \mathrm{mg}_{\text {cat. }}{ }^{-1}(-0.15 \mathrm{~V}$ vs. RHE $)$ & 20.0 & [65] \\
\hline & $\mathrm{Ru}-\mathrm{Mo}_{2} \mathrm{CT}_{\mathrm{x}}$ & $0.5 \mathrm{~mol} / \mathrm{L} \mathrm{K}_{2} \mathrm{SO}_{4}$ & $40.57 \mu \mathrm{g} \cdot \mathrm{h}^{-1} \cdot \mathrm{mg}^{-1}(-0.3 \mathrm{~V}$ vs. RHE $)$ & 25.77 & [38] \\
\hline & $\mathrm{RuSAs} / \mathrm{N}-\mathrm{C}$ & $0.05 \mathrm{~mol} / \mathrm{L} \mathrm{H}_{2} \mathrm{SO}_{4}$ & $120.9 \mu \mathrm{g}_{\mathrm{NH} 3} \cdot \mathrm{mg}_{\text {cat. }}{ }^{-1} \cdot \mathrm{h}^{-1}(-0.3 \mathrm{~V}$ vs. RHE $)$ & 29.6 & [39] \\
\hline \multirow{8}{*}{$\begin{array}{l}\text { 生物固氮酶同 } \\
\text { 组分催化剂 } \\
\text { (Mo、V) }\end{array}$} & $\mathrm{MoS}_{2}$ & $0.1 \mathrm{~mol} / \mathrm{L} \mathrm{Na}_{2} \mathrm{SO}_{4}$ & $8.08 \times 10^{-11} \mathrm{~mol} \cdot \mathrm{s}^{-1} \cdot \mathrm{cm}^{-2}(-0.3 \mathrm{~V}$ vs. RHE $)$ & 1.17 & [59] \\
\hline & $\mathrm{Mo}_{2} \mathrm{~N}$ & $0.1 \mathrm{~mol} / \mathrm{L} \mathrm{HCl}$ & $78.4 \mu \mathrm{g} \cdot \mathrm{h}^{-1} \cdot \mathrm{mg}_{\text {cat. }^{-1}}(-0.3 \mathrm{~V}$ vs. RHE $)$ & 4.5 & [60] \\
\hline & SA-Mo/NPC & $0.1 \mathrm{~mol} / \mathrm{L} \mathrm{KOH}$ & $34.0 \mu \mathrm{g} \cdot \mathrm{h}^{-1} \cdot \mathrm{g}_{\text {cat. }}{ }^{-1}(-0.3 \mathrm{~V}$ vs. RHE $)$ & 14.6 & [43] \\
\hline & $\mathrm{Mo}_{3} \mathrm{Fe}_{3} \mathrm{C}$ & $0.1 \mathrm{~mol} / \mathrm{L} \mathrm{Li}_{2} \mathrm{SO}_{4}$ & $72.5 \mu \mathrm{g} \cdot \mathrm{h}^{-1} \cdot \mathrm{cm}^{-2}(-0.05 \mathrm{~V}$ vs. RHE $)$ & 43.6 & [69] \\
\hline & $\mathrm{V}_{8} \mathrm{C}_{7}$ & $0.1 \mathrm{~mol} / \mathrm{L} \mathrm{HCl}$ & $34.62 \mu \mathrm{g} \cdot \mathrm{h}^{-1} \cdot \mathrm{cm}^{-2}(-0.4 \mathrm{~V}$ vs. RHE $)$ & 12.20 & [45] \\
\hline & $\mathrm{VN}$ & $0.1 \mathrm{~mol} / \mathrm{L} \mathrm{HCl}$ & $2.48 \times 10^{-10} \mathrm{~mol}^{-1} \cdot \mathrm{s}^{-1} \cdot \mathrm{cm}^{-2}(-0.3 \mathrm{~V}$ vs. RHE $)$ & 3.58 & [46] \\
\hline & $\mathrm{VO}_{2}$ & $0.1 \mathrm{~mol} / \mathrm{L} \mathrm{Na}_{2} \mathrm{SO}_{4}$ & $14.85 \mu \mathrm{g} \cdot \mathrm{h}^{-1} \cdot \mathrm{g}_{\text {cat. }}{ }^{-1}(-0.7 \mathrm{~V}$ vs. RHE $)$ & 3.97 & [61] \\
\hline & $\mathrm{P}-\mathrm{V}_{2} \mathrm{O}_{3} / \mathrm{C}$ & $0.1 \mathrm{~mol} / \mathrm{L} \mathrm{Na}_{2} \mathrm{SO}_{4}$ & $22.4 \mu \mathrm{g} \cdot \mathrm{h}^{-1} \cdot \mathrm{mg}_{\text {cat. }}{ }^{-1}(-0.35 \mathrm{~V}$ vs. RHE $)$ & 13.78 & [62] \\
\hline \multirow[t]{5}{*}{ 贵金属催化剂 } & $\mathrm{Au}$ 纳米棒 & $0.1 \mathrm{~mol} / \mathrm{L} \mathrm{KOH}$ & $1.648 \mu \mathrm{g} \cdot \mathrm{h}^{-1} \cdot \mathrm{cm}^{-2}(-0.2 \mathrm{~V}$ vs. RHE $)$ & 4.02 & [50] \\
\hline & $\mathrm{Au}$ & $0.1 \mathrm{~mol} / \mathrm{L} \mathrm{HCl}$ & $21.4 \mu \mathrm{gh}^{-1} \cdot \mathrm{mg}_{\text {cat. }}{ }^{-1}(-0.2 \mathrm{~V}$ vs. RHE $)$ & 8.11 & [23] \\
\hline & 单原子 $\mathrm{Au}$ & $5 \mathrm{mmol} / \mathrm{L} \mathrm{H}_{2} \mathrm{SO}_{4}$ & $1305 \mu \mathrm{g} \cdot \mathrm{h}^{-1} \cdot \mathrm{mg}_{\mathrm{Au}}^{-1}(-0.1 \mathrm{~V}$ vs. RHE $)$ & 11.1 & [51] \\
\hline & 单原子 Pt & $0.1 \mathrm{~mol} / \mathrm{L} \mathrm{K}_{2} \mathrm{SO}_{4}$ & $342.4 \mu \mathrm{g} \cdot \mathrm{h}^{-1} \cdot \mathrm{mg}_{\mathrm{Pt}}{ }^{-1}(-0.2 \mathrm{~V}$ vs. RHE $)$ & 31.3 & [53] \\
\hline & $\mathrm{PdCu}$ & $0.1 \mathrm{~mol} / \mathrm{L} \mathrm{KOH}$ & $2.8 \mu \mathrm{g} \cdot \mathrm{h}^{-1} \cdot \mathrm{mg}_{\text {cat. }}^{-1}(-0.2 \mathrm{~V}$ vs. RHE $)$ & 0.6 & [48] \\
\hline \multirow{6}{*}{$\begin{array}{l}\text { 非贵金属催化 } \\
\text { 剂 }\end{array}$} & $\mathrm{Cu}-\mathrm{TiO}_{2}$ & $0.5 \mathrm{~mol} / \mathrm{L} \mathrm{LiClO}_{4}$ & $21.31 \mu \mathrm{g} \cdot \mathrm{h}^{-1} \cdot \mathrm{mg}_{\text {cat. }}^{-1}(-0.55 \mathrm{~V}$ vs. RHE $)$ & 21.99 & [54a] \\
\hline & Sn 枝晶 & $0.1 \mathrm{~mol} / \mathrm{L}$ PBS & $5.66 \times 10^{-11} \mathrm{~mol}^{-1} \cdot \mathrm{s}^{-1} \cdot \mathrm{cm}^{-2}(-0.6 \mathrm{~V}$ vs. RHE $)$ & 3.67 & [54b] \\
\hline & $\mathrm{Cr}_{2} \mathrm{O}_{3} / \mathrm{rGO}$ & $0.1 \mathrm{~mol} / \mathrm{L} \mathrm{HCl}$ & $33.3 \mu \mathrm{g} \cdot \mathrm{h}^{-1} \cdot \mathrm{mg}_{\text {cat. }}{ }^{-1}(-0.7 \mathrm{~V}$ vs. RHE $)$ & 7.33 & [54c] \\
\hline & $\mathrm{LiMn}_{2} \mathrm{O}_{4}$ & $0.1 \mathrm{~mol} / \mathrm{L} \mathrm{HCl}$ & $15.83 \mu \mathrm{g} \cdot \mathrm{h}^{-1} \cdot \mathrm{mg}_{\text {cat. }}^{-1}(-0.5 \mathrm{~V}$ vs. RHE $)$ & 7.44 & [54d] \\
\hline & $\mathrm{LaFeO}_{3}$ & $0.1 \mathrm{~mol} / \mathrm{L} \mathrm{HCl}$ & $18.59 \mu \mathrm{g} \cdot \mathrm{h}^{-1} \cdot \mathrm{mg}_{\text {cat. }}{ }^{-1}(-0.55 \mathrm{~V}$ vs. RHE $)$ & 8.77 & [54e] \\
\hline & $\mathrm{Bi}$ 纳米片 & $0.1 \mathrm{~mol} / \mathrm{L} \mathrm{HCl}$ & $6.89 \times 10^{-11} \mathrm{~mol}^{-1} \cdot \mathrm{s}^{-1} \cdot \mathrm{cm}^{-2}(-0.5 \mathrm{~V}$ vs. RHE $)$ & 10.26 & [56] \\
\hline
\end{tabular}

比 $\mathrm{N}_{2}$ 还原更容易, HER 与 NRR 间的竞争降低了催化剂 NRR 选择性, 因此如何提升 HER 势垒、减缓 HER 动力 学是函待解决的问题 ${ }^{[63]}$. Lee 等 ${ }^{[64]}$ 提出在 $\mathrm{N}_{2}$ 活化中心表 面包覆疏水层的策略. 通过在 $\mathrm{Ag}-\mathrm{Au}$ 表面修饰疏水的 ZIF 材料(Ag-Au@ZIF)来抑制 $\mathrm{H}^{+}$在催化剂表面吸附, 其 结构示意图和扫描电子显微镜(SEM)如图 9(a), 9(b)所 示. 图 9(c)比较了 Ag-Au@ZIF 和 Ag-Au 催化剂氨合成 法拉第效率, Ag-Au 仅为 $8 \%$, 而 Ag-Au@ZIF 提升至 $18 \%$, 该工作表明在 $\mathrm{N}_{2}$ 活化催化剂表面包覆疏水层可 有效抑制 HER，从而提升产物选择性.

使用低 HER 选择性的材料作为 NRR 催化剂载体是 另一种有效抑制 HER 的策略. Tong 等 ${ }^{[65]}$ 选用 HER 选择 性极低的 $\mathrm{W}_{18} \mathrm{O}_{49}$ 作载体, 构建 $\mathrm{Fe}-\mathrm{W}_{18} \mathrm{O}_{49}$ 催化剂. 利用 $\mathrm{W}_{18} \mathrm{O}_{49}$ 有较低的 $\mathrm{H}$ 结合能, 抑制 HER 在 $\mathrm{Fe}-\mathrm{W}_{18} \mathrm{O}_{49}$ 表 面进行的同时提升了催化剂对 $\mathrm{N}_{2}$ 的吸附活化能力.
$\mathrm{Fe}-\mathrm{W}_{18} \mathrm{O}_{49}$ 催化剂在 $-0.15 \mathrm{~V}$ vs. RHE 电位下合成氨产 率可达 $24.7 \mu \mathrm{g}^{\circ} \mathrm{h}^{-1} \cdot \mathrm{mg}_{\text {cat. }}{ }^{-1}$, 法拉第效率为 $20.0 \%$. 此外, $\mathrm{ZrO}_{2}$ 被证实也可有效抑制 $\mathrm{HER}^{[66]}$, 提升 NRR 选择性. 通过向 $\mathrm{Ru}$ 基催化剂添加 $\mathrm{ZrO}_{2}$, 电催化合成氨法拉第效 率可由 $9 \%$ 提升至 $21 \%$.

将高效的 $\mathrm{N}_{2}$ 活化组分与低析氢选择性的材料耦合 是提升电催化 NRR 效率的有效策略, 该方向的研究还 处于初步阶段. 作为电催化剂的载体, 材料不仅需要具 有低析氢选择性，同时要具有良好的电子传导能力，以 保证电催化反应进行. 此外还需设计合理的催化剂组分 与结构, 使 $\mathrm{N}_{2}$ 活化中心与低 HER 选择性载体高效耦合, 有效提升电催化 NRR 产物选择性.

\section{2 双中心催化剂构建}

除 HER 竞争问题外, $\mathrm{N}_{2}$ 活化、活化 $* \mathrm{~N}$ 加氢效率低 

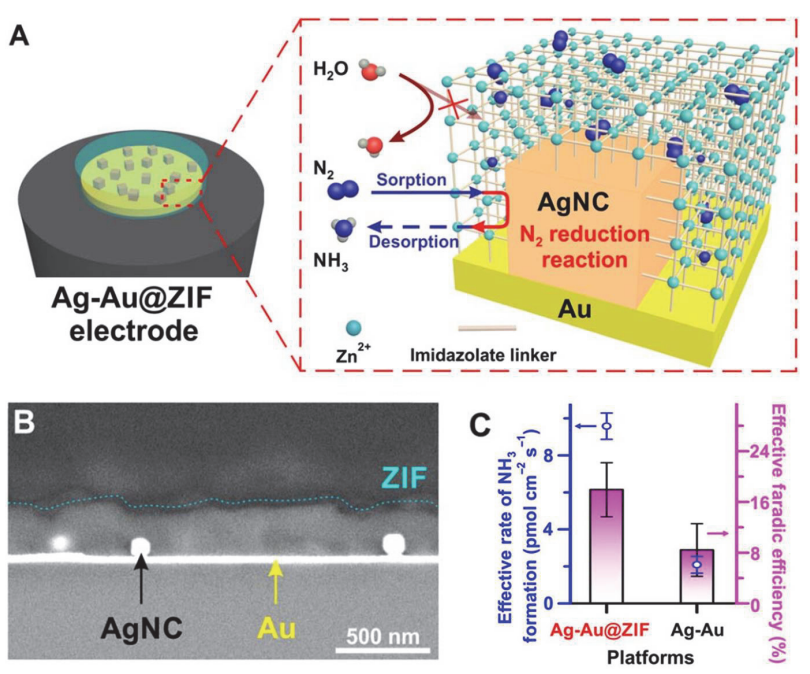

图 9 (A) Ag-Au@ZIF 表面疏水及 NRR 过程示意图; (B) SEM 图; (C) $\mathrm{Ag}-\mathrm{Au} @ \mathrm{ZIF}$ 和 Ag-Au 合成氨产率及法拉第效率

Figure 9 (A) Schematic of Ag-Au@ZIF as water repellent and NRR process; (B) SEM image of Ag-Au@ZIF; (C) ammonia synthesis yield and Faraday efficiency of Ag-Au@ZIF and Ag-Au

也同样制约了催化剂 NRR 性能. Norskov 等 ${ }^{\left[{ }^{[7]}\right.}$ 利用 DFT 计算揭示了催化剂表面 $\mathrm{N}_{2}$ 的吸附与 $\mathrm{N}_{2}$ 活化后的中间态 $\mathrm{N} 、 \mathrm{NH} 、 \mathrm{NH}_{2}$ 等物种的吸附之间存在线性限制, 如图 10 所示. 催化剂对 $\mathrm{N}_{2}$ 有较强吸附, 有利于 $\mathrm{N}_{2}$ 活化, 然而 $\mathrm{N}_{2}$ 活化后与金属成键生成的中间物种过于稳定, 不利 于最终产物脱附. 而催化剂对 $\mathrm{N}_{2}$ 吸附弱, 则不利于 $\mathrm{N}_{2}$ 活化, 这说明具有单一组分的催化剂难以实现温和条件 下高效合成氨.

对于电催化 NRR 催化剂, 其表面不仅要完成 $\mathrm{N}_{2}$ 活 化加氢, 同时要抑制 $\mathrm{H}^{+}$还原, 双金属催化剂可比单金 属催化剂更具有优势 $[68]$. 将对 $\mathrm{N}_{2}$ 活化效率高的材料(对 $\mathrm{N}_{2}$ 吸附能高)与对 HER 选择性低的材料结合可提升催化 剂在常温常压下 NRR 性能. Qin 等 ${ }^{\left[{ }^{[6]}\right.}$ 以四水合钼酸铵、 硝酸铁、甲壳胺、十六烷基三甲基溴化铵为原料, 通过 水热-热解的方式制备 $\mathrm{Mo}_{3} \mathrm{Fe}_{3} \mathrm{C}$ 催化剂. DFT计算结果表

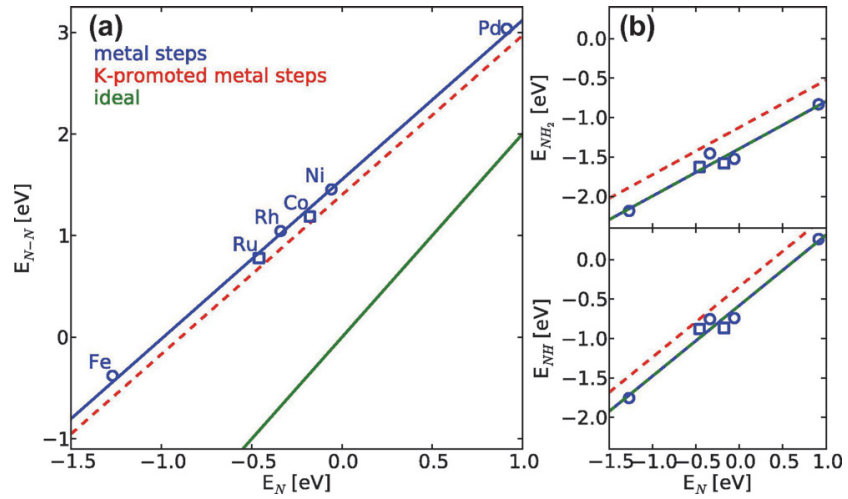

图 10 (a)过渡金属表面 $\mathrm{N}$ 的吸附能与 $\mathrm{N}_{2}$ 解离势垒之间的线性关系; (b)过渡金属表面 $\mathrm{NH}$ 和 $\mathrm{NH}_{2}$ 吸附能之间的线性关系

Figure 10 (a) Calculated transition state energies for $\mathrm{N}_{2}$ dissociation as a function of the nitrogen adsorption energy over step site; (b) adsorption energy scaling relations for $\mathrm{NH}$ and $\mathrm{NH}_{2}$ adsorbed intermediates
明, $\mathrm{Fe}$ 的引入可以提升 $\mathrm{Mo}$ 对 $\mathrm{N}_{2}$ 活化加氢能力, $\mathrm{Fe}$ 与 Mo 可产生协同作用, 共同活化 $\mathrm{N}_{2}$ 分子. 当 $\mathrm{N}_{2}$ 在 $\mathrm{Mo}_{2} \mathrm{C}(121), \mathrm{Fe}_{3} \mathrm{C}(111), \mathrm{Mo}_{3} \mathrm{Fe}_{3} \mathrm{C}(111)$ 表面发生吸附后, $\mathrm{N}_{2}$ 分子键长由 $0.1110 \mathrm{~nm}$ 分别拉长至 $0.1132,0.1127$, $0.1195 \mathrm{~nm}$ ，这表明 $\mathrm{Mo}_{2} \mathrm{C}(121), \mathrm{Fe}_{3} \mathrm{C}(111), \mathrm{Mo}_{3} \mathrm{Fe}_{3} \mathrm{C}(111)$ 面均可活化 $\mathrm{N}_{2}$, 其中 $\mathrm{Mo}_{3} \mathrm{Fe}_{3} \mathrm{C}(111)$ 对 $\mathrm{N}_{2}$ 活化能力最强. 图 11(a) 11(c) 计算了 $\mathrm{N}_{2}$ 分别在 $\mathrm{Mo}_{2} \mathrm{C}(121)$, $\mathrm{Mo}_{3} \mathrm{Fe}_{3} \mathrm{C}(111), \mathrm{Fe}_{3} \mathrm{C}(111)$ 面活化加氢过程中相对势能 $(\Delta E)$ 变化, 结果显示 $* \mathrm{~N}_{2}$ 在 $\mathrm{Mo}_{3} \mathrm{Fe}_{3} \mathrm{C}(111)$ 面加氢生成 $* \mathrm{~N}_{2} \mathrm{H}$ 所需势垒明显低于 $\mathrm{Mo}_{2} \mathrm{C}(121), \mathrm{Fe}_{3} \mathrm{C}(111)$ 面. NRR 活性测试验证了这一结果, $\mathrm{Mo}_{3} \mathrm{Fe}_{3} \mathrm{C}$ 催化剂合成氨速率 为 $72.5 \mu \mathrm{g} \cdot \mathrm{h}^{-1} \cdot \mathrm{cm}^{-2}$, 法拉第效率高达 $43.6 \%$. 而 $\mathrm{Mo}_{2} \mathrm{C}$ 催化剂合成氨速率为 $50.1 \mu \mathrm{g} \cdot \mathrm{h}^{-1} \cdot \mathrm{cm}^{-2}$, 法拉第效率仅 为 3.9\%(图 11(d), 11(e)). 构建双中心催化剂是提升常温 常压 NRR 性能的有效策略, 将两种活性组分的特点有 效耦合，可使其在 NRR 领域中表现出独特的优势，目前 在篎选合理的催化剂组分、精准构建催化剂结构方面还 需进行大量的实践工作.

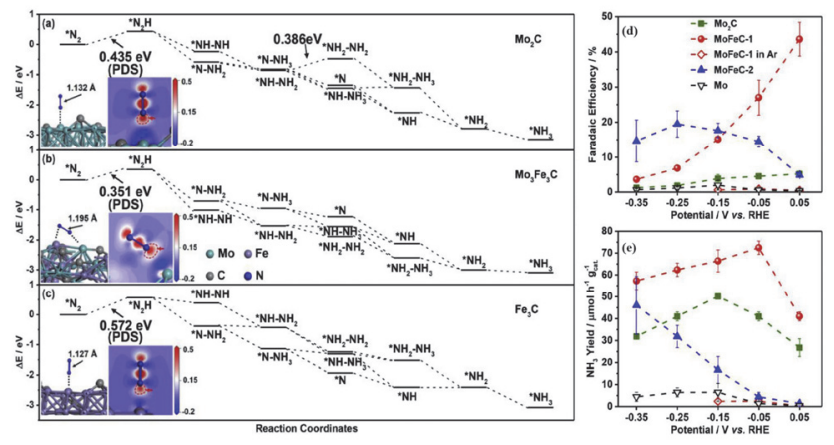

图 11 分别在(a) $\mathrm{Mo}_{2} \mathrm{C}(121)$, (b) $\mathrm{Mo}_{3} \mathrm{Fe}_{3} \mathrm{C}(111)$, (c) $\mathrm{Fe}_{3} \mathrm{C}(111)$ 面, $\mathrm{NRR}$ 过程中可能的中间产物相对势能 $(\Delta E)$ 变化图; (d)催化剂合成氨法拉第 效率和(e)产率图

Figure 11 DFT calculation results of relative potential energies $(\Delta E)$ for possible intermediates during NRR on (a) $\mathrm{Mo}_{2} \mathrm{C}$, (b) $\mathrm{Mo}_{3} \mathrm{Fe}_{3} \mathrm{C}$, (c) $\mathrm{Fe}_{3} \mathrm{C}$; (d) FEs and (e) $\mathrm{NH}_{3}$ yield of NRR on different samples under different potential

\section{3 添加助剂}

在工业合成氨过程中, 为提升反应效率、稳定性, 通常向催化剂中添加少量助剂 ${ }^{[70]}$. 按照助剂的作用, 可 分为结构助剂和电子助剂. 结构助剂的作用可提升催化 剂分散性，防止活性组分的晶粒在反应过程中长大，热 催化研究中常用的结构助剂有 $\mathrm{Al}_{2} \mathrm{O}_{3} 、 \mathrm{MgO}$ 等. 电子助 剂(如 $\mathrm{K}$ 和 $\mathrm{Cs}$ ) 可影响催化剂电子转移能力, 提升活性中 心催化活性、产物选择性. 对于常温常压液相 NRR研究, 添加电子助剂被报道可显著提升 NRR 活性. Hao 等 ${ }^{[71]}$ 研究了 $\mathrm{K}^{+}$作为助剂对电催化合成氨活性、产物选择性 的影响. 如图 12 所示, 当电解液中 $\mathrm{K}^{+}$离子浓度从 0.2 提升到 $1.0 \mathrm{~mol} / \mathrm{L}, \mathrm{Bi}$ 基催化剂 $\mathrm{NRR}$ 电流密度从 0.14 增 长到 $0.50 \mathrm{~mA} \cdot \mathrm{cm}^{-2}$, 法拉第效率从 $9.8 \%$ 增长到 $66 \%$, 合 成氨产率可达 $200 \mathrm{mmol} \cdot \mathrm{g}^{-1} \cdot \mathrm{h}^{-1}$. DFT 计算结果显示 $\mathrm{K}^{+}$ 离子引入可降低 $\mathrm{N}_{2}$ 在 $\mathrm{Bi}(012) 、(110)$ 晶面还原反应势垒, 
提升 $\mathrm{N}_{2}$ 活化效率. 需要注意的是, 电子助剂对电催化 NRR 性能的影响还存在争议, 对于电子助剂的真实作 用需进一步被证实. 此外, 结构助剂对电催化 NRR 的影 响还鲜有研究.
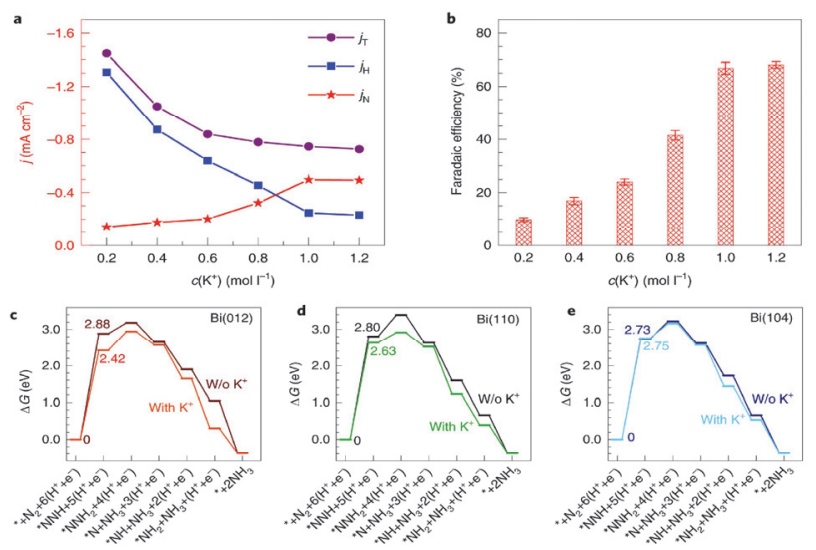

图 12 (a) $\mathrm{Bi}$ 基催化剂在不同 $\mathrm{K}^{+}$离子浓度下 $\mathrm{N}_{2}$ 还原电流密度 $\left(j_{\mathrm{N}}\right)$, HER 电流密度 $\left(j_{\mathrm{H}}\right)$ 和总反应电流密度 $\left(j_{\mathrm{T}}\right)$ 图; (b) 在不同 $\mathrm{K}^{+}$离子浓度下 $\mathrm{NRR}$ 法拉第效率图; (c) (e) 有 $\mathrm{K}^{+}$或无 $\mathrm{K}^{+}$离子, $\mathrm{Bi}(012),(110),(104)$ 晶面在电催化 $\mathrm{N}_{2}$ 还原至 $\mathrm{NH}_{3}$ 过程中的吉布斯自由能图

Figure 12 Promotion of ENRR on BiNCs by potassium cations. (a) NRR current density $\left(j_{\mathrm{N}}\right)$, HER current density $\left(j_{\mathrm{H}}\right)$ and total current density $\left(j_{\mathrm{T}}\right)$; (b) ENRR Faradaic efficiency versus $c\left(\mathrm{~K}^{+}\right)$; (c) $\sim(\mathrm{e})$ Gibbs free-energy diagrams of the electrochemical reduction of $\mathrm{N}_{2}$ to $\mathrm{NH}_{3}$ on $\mathrm{Bi}(012),(110)$ and (104) facets in the presence or absence of $\mathrm{K}^{+}$

\section{$5 \mathrm{NO}_{3}{ }^{-}$、 $\mathrm{NO}$ 还原至 $\mathrm{NH}_{3}$ 研究进展}

\section{$5.1 \mathrm{NO}_{3}{ }^{-}$还原}

相较于 $\mathrm{N}_{2}, \mathrm{NO}_{3}{ }^{-}$还原制 $\mathrm{NH}_{3}$ 在热力学上更容易, 利 用地表水和地下水中大量存在的 $\mathrm{NO}_{3}{ }^{-}$合成氨对环保和 节能方面都有重要意义 ${ }^{[72]}$. 虽然 $\mathrm{NO}_{3}{ }^{-}$还原在热力学上 比 $\mathrm{N}_{2}$ 还原更容易实现, 但 $\mathrm{NO}_{3}{ }^{-}$还原到 $\mathrm{NH}_{3}$ 涉及到 $8 \mathrm{e}^{-}$ 转移, 催化剂在热力学上更倾向于通过 $5 \mathrm{e}^{-}$将 $\mathrm{NO}_{3}{ }^{-}$还原 成 $\mathrm{N}_{2}$. 此外, 与 $\mathrm{N}_{2}$ 还原相同, $\mathrm{NO}_{3}{ }^{-}$还原也面临 HER 竞 争. $\mathrm{NO}_{3}{ }^{-}$还原到 $\mathrm{NH}_{3}$ 的反应式见式(4).

$\mathrm{NO}_{3}{ }^{-}+9 \mathrm{H}^{+}+8 \mathrm{e}^{-} \rightarrow \mathrm{NH}_{3}+3 \mathrm{H}_{2} \mathrm{O} \quad E=1.20 \mathrm{~V}$ vs. NHE (4)

实际上 $\mathrm{NO}_{3}{ }^{-}$还原的过程非常复杂, $\mathrm{N}$ 的价态从 +5 价变为一 -3 价, 在还原过程中, $\mathrm{NO}_{3}{ }^{-}$首先被还原为 $\mathrm{NO}_{2}{ }^{-}$, 随后还原成 $\mathrm{NO}, \mathrm{NO}$ 进一步加氢生成 $\mathrm{HNO} 、 \mathrm{H}_{2} \mathrm{NO}$, 最 终形成 $\mathrm{NH}_{3}{ }^{[73]}$. 还原 $\mathrm{NO}_{3}{ }^{-}$的关键在于, 催化剂中金属 的最高占据 $\mathrm{d}$ 轨道与 $\mathrm{NO}_{3}{ }^{-}$的 LUMO 能级匹配, 以促进 金属 $\mathrm{d}$ 轨道的 $\mathrm{e}^{-}$向 $\mathrm{NO}_{3}{ }^{-}$的 LUMO 能级转移. 现阶段的 研究表明, $\mathrm{Cu} 、 \mathrm{Ru}$ 基催化剂的 $\mathrm{NO}_{3}{ }^{-}$还原制 $\mathrm{NH}_{3}$ 选择性 较高, 而 $\mathrm{Fe} 、 \mathrm{Ni} 、 \mathrm{Co}$ 等金属更倾向于将 $\mathrm{NO}_{3}{ }^{-}$还原至 $\mathrm{N}_{2}{ }^{[74]}$. Wang 等 ${ }^{[75]}$ 利用阳极氧化在 $\mathrm{Cu}$ 基底上生长 $\mathrm{Cu}(\mathrm{OH})_{2}$ 纳米阵列, 随后 $\mathrm{Cu}(\mathrm{OH})_{2}$ 在 $\mathrm{O}_{2}$ 气气氛中进行热 处理, 得到 $\mathrm{CuO}$ 纳米阵列. 在电化学测试中 $\mathrm{CuO}$ 最终转 换为 $\mathrm{Cu} / \mathrm{Cu}_{2} \mathrm{O}$, 通过对比实验证明 $\mathrm{Cu} / \mathrm{Cu}_{2} \mathrm{O}$ 是 $\mathrm{NO}_{3}{ }^{-}$还 原的有效组分. 在 $\mathrm{NO}_{3}{ }^{-}$浓度为 $200 \mathrm{mg} / \mathrm{L}$ 的 $0.5 \mathrm{~mol} / \mathrm{L}$ $\mathrm{Na}_{2} \mathrm{SO}_{4}$ 电解液中, $\mathrm{Cu} / \mathrm{Cu}_{2} \mathrm{O}$ 催化剂还原 $\mathrm{NO}_{3}{ }^{-}$生成 $\mathrm{NH}_{3}$
产率高达 $0.24 \mathrm{mmol} \cdot \mathrm{h}^{-1} \cdot \mathrm{cm}^{-1}$, 转化率为 $81.2 \%$, 法拉第 效率为 $95.8 \%$. 使用 $\mathrm{Na}^{15} \mathrm{NO}_{3}$ 进行同位素实验, 证实了 $\mathrm{NH}_{3}$ 的生成来自 $\mathrm{NO}_{3}{ }^{-}$还原, DFT 计算表明, $\mathrm{Cu} / \mathrm{Cu}_{2} \mathrm{O}$ 可 降低 HNO 的生成势垒，同时还可有效抑制 HER 竞争(图 13). $\mathrm{Li}$ 等 ${ }^{[76]}$ 设计了具有 core-shell 结构的 $\mathrm{Ru} / \mathrm{RuO}_{2}$ 纳米 团簇, $\mathrm{Ru}$ 颗粒的平均尺寸为 $2 \mathrm{~nm}$, 氨的转化率接近 $100 \%$, 产率为 $5.56 \mathrm{~mol} \cdot \mathrm{g}_{\text {cat. }}{ }^{-1} \cdot \mathrm{h}^{-1}$.

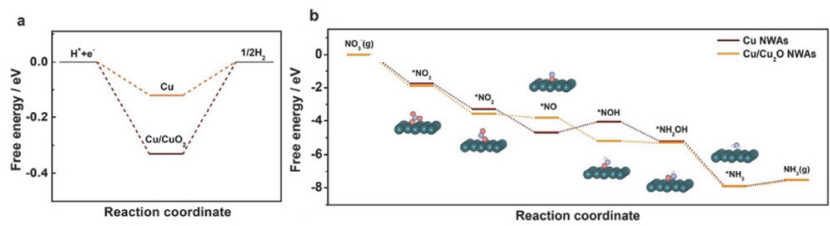

图 13 (a) $\mathrm{H}_{2}$ 分别在 $\mathrm{Cu}$ NWAs 和 $\mathrm{Cu} / \mathrm{Cu}_{2} \mathrm{O}$ NWAs 表面生成反应能; (b) $\mathrm{Cu}$ NWAs 和 $\mathrm{Cu} / \mathrm{Cu}_{2} \mathrm{O}$ NWAs 合成氨过程自由能图

Figure 13 (a) The reaction energies of $\mathrm{H}_{2}$ formation over $\mathrm{Cu} / \mathrm{Cu}_{2} \mathrm{O}$ NWAs and Cu NWAs; (b) free energy diagram for NRA over $\mathrm{Cu}$ NWAs and $\mathrm{Cu} / \mathrm{Cu}_{2} \mathrm{O}$ NWAs

开发高效的 $\mathrm{NO}_{3}{ }^{-}$还原制 $\mathrm{NH}_{3}$ 催化剂对常温常压氨 合成研究具有重要意义. 上述的实验工作表明在高浓度 的 $\mathrm{NO}_{3}{ }^{-}$环境中, 可实现高效、高选择性氨合成, 但实际 上水中的 $\mathrm{NO}_{3}{ }^{-}$浓度远低于实验条件, 大量的 $\mathrm{H}^{+}$将占据 $\mathrm{NO}_{3}{ }^{-}$还原活性位点, 电催化 $\mathrm{NO}_{3}{ }^{-}$还原同样面临着严重 的 HER 竞争, 因此在催化剂设计方面还需要考虑如何 降低 HER 竞争的影响.

$\mathrm{NO}_{3}{ }^{-}$还原的研究可为 $\mathrm{N}_{2}$ 还原到 $\mathrm{NH}_{3}$ 的工作提供借 鉴. Wang 等 ${ }^{[77]}$ 提出先将空气中的 $\mathrm{N}_{2}$ 氧化为 $\mathrm{NO}_{3}{ }^{-}$, 再还 原到 $\mathrm{NH}_{3}$ 的路径. 以 $0.3 \mathrm{~mol} / \mathrm{L} \mathrm{K}_{2} \mathrm{SO}_{4}$ 为电解液, $\mathrm{Pt}$ 片为 工作电极, 当施加电位高于 $2.13 \mathrm{~V}$ vs. RHE, 可将空气 中的 $\mathrm{N}_{2}$ 氧化为 $\mathrm{NO}_{3}{ }^{-}$和 $\mathrm{NO}_{2}{ }^{-}$, 这一过程的法拉第效率为 $1.23 \%$. 随后以 $\mathrm{Co}_{3} \mathrm{O}_{4}$ 为 $\mathrm{NO}_{3}{ }^{-}$还原催化剂, 在 $-0.65 \mathrm{~V}$ vs. RHE 电位下, $\mathrm{NH}_{3}$ 合成选择性为 $33.6 \%$.

\section{$5.2 \mathrm{NO}$ 还原}

NO 是一种无色无味, 难溶于水的有毒气体，是大 气中的主要污染源之一. NO 的主要来源有工业生产产 生的烟气与汽车尾气. NO 在空气中会转换成酸雨危害 环境, 还可破坏大气的臭氧层 ${ }^{[78]}$. 选择性催化还原法 (SCR) 是目前消除 NO 的最普遍的方法, 利用催化剂和 还原剂(如氨气, 液氨)与 $\mathrm{NO}$ 反应生成无毒无害的 $\mathrm{N}_{2}$ 和 $\mathrm{H}_{2} \mathrm{O}$, 其反应式如下 ${ }^{[79]}$ :

$$
\begin{aligned}
& 4 \mathrm{NO}+4 \mathrm{NH}_{3}+\mathrm{O}_{2} \rightarrow 4 \mathrm{~N}_{2}+6 \mathrm{H}_{2} \mathrm{O} \\
& 6 \mathrm{NO}+4 \mathrm{NH}_{3} \rightarrow 5 \mathrm{~N}_{2}+6 \mathrm{H}_{2} \mathrm{O} \\
& 2 \mathrm{NO}+2 \mathrm{NO}_{2}+4 \mathrm{NH}_{3} \rightarrow 4 \mathrm{~N}_{2}+6 \mathrm{H}_{2} \mathrm{O}
\end{aligned}
$$

但该技术需要消耗大量的 $\mathrm{NH}_{3}$, 因此这一技术成本 较高, 同时能源消耗大. 鉴于此, Long 等 ${ }^{[80]}$ 提出将 $\mathrm{NO}$ 直接还原至 $\mathrm{NH}_{3}$, 这一过程不仅可避免 $\mathrm{NH}_{3}$ 的消耗, 还 可将 NO 转换成能源进行储存. 基于 DFT 计算, 研究人 员讨论了 $\mathrm{NO}$ 分别在 $\mathrm{V} 、 \mathrm{Cr} 、 \mathrm{Mo} 、 \mathrm{Ru} 、 \mathrm{Pd} 、 \mathrm{Pt} 、 \mathrm{Cu} 、$ 
$\mathrm{Ag} 、 \mathrm{Au}$ 表面合成氨反应路径, 结果表明 $\mathrm{NO}$ 还原至 $\mathrm{NH}_{3}$ 在热力学上比 $\mathrm{N}_{2}$ 还原更容易. 图 14 是 $\mathrm{Cu}(111)$ 面的 HER 以及 $\mathrm{NO}$ 还原到 $\mathrm{NH}_{3} 、 \mathrm{~N}_{2} \mathrm{O}$ 和 $\mathrm{N}_{2}$ 反应的自由能变化图. 在 $\mathrm{Cu}(111)$ 表面, $\mathrm{NO}$ 还原至 $\mathrm{NH}^{*} 、 \mathrm{~N}_{2} \mathrm{O}$ 和 $\mathrm{N}_{2}$ 的反应势 垒分别为 $0.42 、 0.60$ 和 $0.92 \mathrm{eV}$, 低于 HER 势垒 $(0.98 \mathrm{eV})$, 表明在 $\mathrm{Cu}(111)$ 面可有效抑制 HER, NO 更倾向还原至 $\mathrm{NH}_{3} . \mathrm{NO}$ 在 $\mathrm{Cu}$ 表面还原成氨的反应式可总结为:

$$
\begin{aligned}
& \mathrm{NO}(\mathrm{g})+\left(\mathrm{H}^{+}+\mathrm{e}^{-}\right) \rightarrow \mathrm{NOH}^{*} \\
& \mathrm{NOH}^{*}+\left(\mathrm{H}^{+}+\mathrm{e}^{-}\right) \rightarrow \mathrm{N}^{*}+\mathrm{H}_{2} \mathrm{O} \\
& \mathrm{N}^{*}+\left(\mathrm{H}^{+}+\mathrm{e}^{-}\right) \rightarrow \mathrm{NH}^{*} \\
& \mathrm{NH}^{*}+\left(\mathrm{H}^{+}+\mathrm{e}^{-}\right) \rightarrow \mathrm{NH}_{2} * \\
& \mathrm{NH}_{2}^{*}+\left(\mathrm{H}^{+}+\mathrm{e}^{-}\right) \rightarrow \mathrm{NH}_{3}^{*}(\mathrm{~g})
\end{aligned}
$$

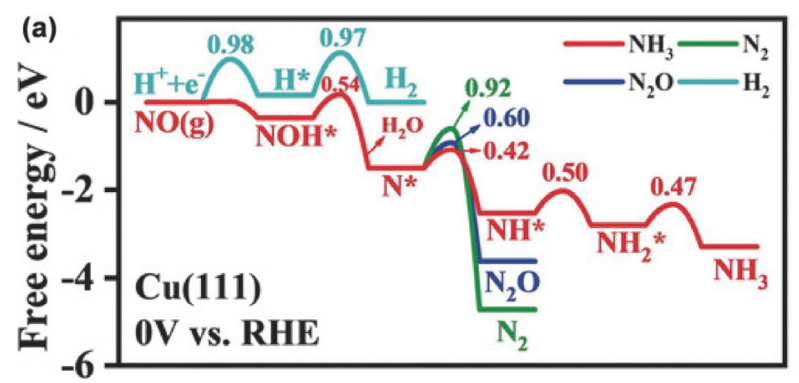

\section{Reaction Coordinate}
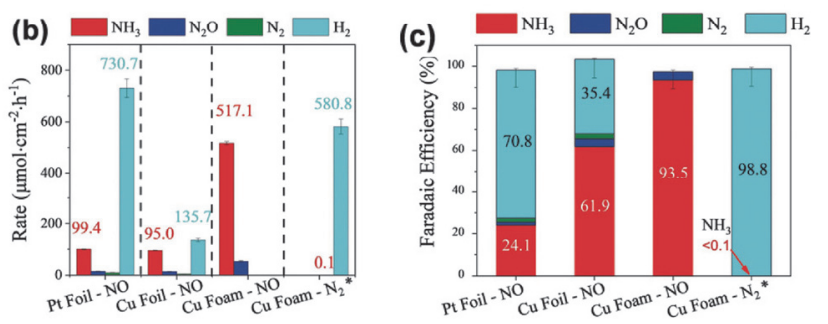

图 14 (a) $\mathrm{Cu}(111)$ 面 $\mathrm{HER}$ 和 $\mathrm{NO}$ 还原到 $\mathrm{NH}_{3}, \mathrm{~N}_{2} \mathrm{O}, \mathrm{N}_{2}$ 反应的自由能 变化图; (b), (c)为在 $\mathrm{Pt}$ 片、 $\mathrm{Cu}$ 片、泡沫 $\mathrm{Cu}$ 电极 $\mathrm{NO}$ 还原到氨的反应 速率和法拉第效率图

Figure 14 (a) Free-energy diagrams for HER, NORR to $\mathrm{NH}_{3}, \mathrm{~N}_{2} \mathrm{O}$ and $\mathrm{N}_{2}$ over $\mathrm{Cu}(111)$; (b) reaction rate and (c) Faradaic efficiency for NORR on a $\mathrm{Pt}$ foil, $\mathrm{Cu}$ foil and $\mathrm{Cu}$ foam

电化学测试使用 $\mathrm{H}$ 型电解池, 中间以 Nafion 115 隔 开, 电解液为 $0.25 \mathrm{~mol} / \mathrm{L}$ 的 $\mathrm{Li}_{2} \mathrm{SO}_{4}, \mathrm{NO}$ 流速为 30 $\mathrm{mL} / \mathrm{min}$. 在电位为 $-0.9 \mathrm{~V}$ vs. RHE 时, $\mathrm{Pt}$ 片, $\mathrm{Cu}$ 片和 $\mathrm{Cu}$ 网还原 $\mathrm{NO}$ 到 $\mathrm{NH}_{3}$ 的反应速率分别为 $99.4,135.7$ 和 580.8 $\mu \mathrm{mol} \cdot \mathrm{h}^{-1} \cdot \mathrm{cm}^{-2}$, 对应的法拉第效率为 $24.1 \%, 61.9 \%$ 和 93.5\%. 氨产率达到了传统 Haber-Bosch 过程产率的量 级, 且电极经 $100 \mathrm{~h}$ 连续测试后, 活性无衰减. 经同位素 标记实验验证, $\mathrm{NH}_{3}$ 全部来源于 $\mathrm{NO}$ 的还原.

对于常温常压合成氨研究, 将大气或水中的不同氮 源转化为氨具有重要的意义与价值. 虽然相关工作还处 于起始阶段, 但已表现出巨大的潜力. 对于 $\mathrm{NO}_{3}{ }^{-}$和 $\mathrm{NO}$ 还原而言, 现阶段需要大量篮选材料, 确定适宜的活性 组分, 以提升氨生成选择性.

\section{6 结论与展望}

开展常温常压合成氨路线对社会发展具有重要意 义. 利用太阳能实现氨的合成有巨大的发展潜力, 一旦 突破将成为能源领域里里程碑式的技术革新. 目前太阳 能催化合成氨领域已经取得了一定进展，但整体效率依 旧偏低, 最主要的原因在于缺少高效的合成氨助催化 剂，开发具有高法拉第效率的 NRR 电催化剂是实现高 效太阳能合成氨的关键. 针对这一现状, 本综述重点介 绍了 NRR 电催化剂研究进展, 提升 NRR 性能的方法策 略, 以及其它氮源 $\left(\mathrm{NO}_{3}{ }^{-} 、 \mathrm{NO}\right)$ 电催化合成氨研究现状. 基于对研究现状的分析, 我们认为还可从以下方面进行 深入研究:

(1)对常温常压 NRR 催化机理认知. 认知催化反应 机理可为设计高效催化剂提供指导. 常温常压 NRR 过 程反应机制复杂，且 $* \mathrm{~N} 、 * \mathrm{NH}$ 、 $* \mathrm{NH}_{2}$ 等中间物种难以 被激光电离, 现阶段对于机理的认知主要是通过理论计 算, 缺乏实验数据, 而理论计算是基于理想条件, 并不 能准确反应催化机制. 因此需发展激光技术, 并将其与 原位 X 射线光电子能谱、原位红外等技术结合, 准确认 知 NRR 机理, 为常温常压 $\mathrm{N}_{2}$ 还原提供科学依据. (2)设 计结构合理的 NRR 电催化剂. $\mathrm{N}_{2}$ 活化困难与 HER 竞争 是常温常压条件下, 液相 NRR 面临的最主要问题. 研究 表明 NRR 催化剂是结构敏感的催化剂，通过精准合成 调控活性中心的结构, 并结合低 HER 材料策略和电子 助剂等方式, 能有效提升催化剂 NRR 活性及选择性. (3) 设计有效的光催化剂与电催化剂耦合体系. 光催化材料 的捕光效率、电荷分离效率、光/电催化剂界面电荷传输 效率同样是决定光催化 NRR 效率的因素. 虽然光催化 合成氨催化剂在捕光材料设计、电荷分离策略等方面可 以直接从光催化水分解得到借鉴 ${ }^{[81]}$, 但如何构建具有 高效电荷传输的界面仍具有挑战，需要通过精准设计合 成实现光催化材料与电催化材料界面有效连接, 从而实 现高效光催化 NRR. (4)不同氮源光催化 NRR. 从热力学 上, $\mathrm{NO}_{3}{ }^{-}$、 $\mathrm{NO}$ 还原比 $\mathrm{N}_{2}$ 还原合成氨更容易, 将水体中 的 $\mathrm{NO}_{3}{ }^{-}$与大气中的 $\mathrm{NO}$ 还原至 $\mathrm{NH}_{3}$, 无论在环境方面还 是能源方面都具有重要意义. 但是 $\mathrm{NO}_{3}{ }^{-}$、 $\mathrm{NO}$ 还原至 $\mathrm{N}_{2}$ 和 $\mathrm{NH}_{3}$ 为竞争反应, 因此需要设计合理的催化剂, 实 现 $\mathrm{NH}_{3}$ 高选择性合成.

\section{作者简介}

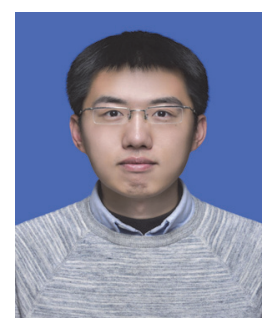

詹溯, 2017 年于大连海事大学载运工具运用工程专业获 得工学博士学位, 2018 年加入中国科学院大连化学物理研究 
所章福祥课题组, 目前主要研究方向为常温常压光电催化合 成氨.

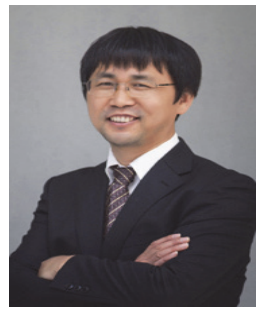

章福祥, 中国科学院大连化物所研究员/博导; 国家杰出 青年基金获得者; 英国皇家化学会会士. 1999 和 2004 年分别 获得南开大学理学学士学位和博士学位, 同年留校任教至 2007 年 8 月, 2007 年 9 月至 2008 年 6 月获法国 CNRS 博士后 基金支持于巴黎第六大学做访问学者, 2008 年 7 月至 2011 年 9 月在东京大学做博士后和特任助理教授, 2011 年 10 月至今 在中国科学院大连化学物理研究所工作. 目前主要从事宽光 谱捕光催化剂全分解水制氢研究, 研究内容涉及宽光谱捕光 光催化材料设计合成, 高效光生电荷分离体系构建以及光催 化表面/界面反应机制等方面. 已在包括 Nat. Commun., Nature Catal., Joule, J. Am. Chem. Soc., Angew. Chem. Int. Ed., Adv. Mater, Adv. Energy Mater. 等刊物上发表学术论文百余篇.

\section{References}

[1] (a) Erisman, J. W.; Sutton, M. A.; Galloway J.; Klimont, Z.; Winiwarter, W. Nat. Geosci. 2008, 1, 636. (b) Ertl, G. Angew. Chem. Int. Ed. 2008, 47, 3524.

[2] (a) Li, X. B.; Tung, C. H.; Wu, L. Z. Angew. Chem. Int. Ed. 2019, 58, 10804. (b) Zamfirescu, C.; Dincer, I. J. Power Sources 2008, 185, 459. (c) Foster, S. L.; Perez Bakovic, S. I.; Duda, R. D.; Maheshwari, S.; Milton, R. D.; Minteer, S. D.; Janik, M. J.; Renner, J. N.; Greenlee, L. F. Nat. Catal. 2018, 1, 490. (d) Guo, J. P.; Chen, P. Chem 2017, 3, 709.

[3] Schuth, F.; Palkovits, R.; Schlogl, R.; Su, D. S. Energy Environ. Sci. 2012, 5, 6278

[4] Liu, H.; Chin, J. J. Catal. 2014, 35, 1619

[5] (a) Rafiqul, I.; Weber, C.; Lehmann, B.; Voss, A. Energy 2005, 30, 2487. (b) Qiu, W. B.; Xie, X. Y.; Qiu, J. D.; Fang, W. H.; Liang, R. P.; Ren, X.; Ji, X. Q.; Cui, G. W.; Asiri, A. M.; Cui, G. L.; Tang, B.; Sun, X. P. Nat. Commun. 2018, 9, 3485.

[6] (a) Nishibayashi, Y.; Saito, M.; Uemura, S.; Takekuma, S.; Takekuma, H.; Yoshida, Z. Nature 2004, 428, 279. (b) Li, H.; Shang, J.; Ai, Z.; Zhang, L. J. Am. Chem. Soc. 2015, 137, 6393. (c) Hao, Y.; Dong, X.; Zhai, S.; Ma, H.; Wang, X.; Zhang, X. Chem. Eur. J. 2016, 22, 18722. (d) Schrock, R. R. Acc. Chem. Res. 2005, 38, 955. (e) Sun, S.; An, Q.; Wang, W.; Zhang, L.; Liu, J.; Goddard, W. A. J. Mater. Chem. A 2017, 5, 201. (f) Sun, S.; Li, X.; Wang, W.; Zhang, L.; Sun, X. Appl. Catal. B-Environ. 2017, 200, 323. (g) Banerjee, A.; Yuhas, B. D.; Margulies, E. A.; Zhang, Y.; Shim, Y.; Wasielewski, M. R.; Kanatzidis, M. G. J. Am. Chem. Soc. 2015, 137, 2030. (h) Liu, J.; Kelley, M. S.; Wu, W.; Banerjee, A.; Douvalis, A. P.; Wu, J.; Zhang, Y.; Schatz, G. C.; Kanatzidis, M. G. Proc. Natl. Acad. Sci. U. S. A. 2016, 113, 5530. (i) Oshikiri, T.; Ueno, K.; Misawa, H. Angew. Chem. Int. Ed. 2014, 53, 9802. (j) Pickett, C. J.; Talarmin, J. Nature 1985, 317, 652. (k) Kordali, V.; Kyriacou, G.; Lambrou, C. H. Chem. Commun. 2000, 0, 1673. (1) Yu, X. M.; Han, P.; Wei, Z. X.; Huang, L. S.; Gu, Z. X.; Peng, S. J.; Ma, J. M.; Zheng, G. F. Joule 2018, 2, 1610. (m) Lv, C.; Qian, Y. M.; Yan, C. S.; Ding, Y.; Liu, Y. Y.; Chen, G.; Yu, G. H. Angew. Chem. Int. Ed. 2018, 57, 10246. (n) Zhang, L. L.; Ding, L. X.; Chen, G. F.; Yang, X. F.; Wang, H. H. Angew. Chem. Int. Ed. 2019, 131, 2612.

[7] Walter, M. G.; Warren, E. L.; Mckone, J. R.; Boettcher, S. W.; Mi, Q. X.; Santori, E. A.; Lewis, N. S. Chem. Rev. 2010, 110, 6446.

[8] Chen, J. G.; Crooks, R. M.; Seefeldt, L. C.; Bren, K. L.; Bullock, R. M.; Darensbourg, M. Y.; Holland, P. L.; Hoffman, B.; Janik, M. J.; Jones, A. K.; Kanatzidis, M. G.; King, P.; Lancaster, K. M.; Lymar,
S. V.; Pfromm, P.; Schneider, W. F.; Schrock, R. R. Science 2018 , 360,873 .

[9] Shilov, A. E. Russ. Chem. Bull. 2003, 52, 2555.

[10] (a) Singh, A. R.; Rohr, B. A.; Schwalbe, J. A.; Cargnello, M.; Chan, K.; Jaramillo, T. F.; Chorkendorff, I. B.; Norskov, J. K. ACS Catal. 2017, 7, 106. (b) Skulason, E.; Bligaard, T.; Gudmundsdottir, S.; Studt, F.; Rossmeisl, J.; Abild-Pedersen, F.; Vegge, T.; Jonsson, H.; Norskov, J. K. Phys. Chem. Chem. Phys. 2012, 14, 1235.

[11] (a) Shipman, M. A.; Symes, M. D. Catal. Today 2017, 286, 57. (b) $\mathrm{Xu}, \mathrm{H}$.; Inthisuphalap, K.; Li, Y.; Mukherjee, S.; Lattimer, J.; Soloveichik, G.; Wu, G. Nano Energy 2020, 69, 104469.

[12] Urabe, K.; Aika, K. I.; Ozaki, A. J. Catal. 1974, 11, 108.

[13] Ithisuphalap, K.; Zhang, H.; Guo, L.; Yang, Q.; Yang, H.; Wu, G. Small Methods 2019, 3, 1800352.

[14] Huang, Y. W.; Zhang, N.; Wu, Z. J.; Xie, X. Q. J. Mater. Chem. A 2020, $8,4978$.

[15] (a) Furuya, N.; Yoshiba, H. J. Electroanal. Chem. Interfacial Electrochem. 1989, 263, 171. (b) Lan, R.; Irvine, J. T. S.; Tao, S. Sci. Rep. 2013, 3, 1145.

[16] (a) Kugler, K.; Luhn, M.; Schramm, J. A.; Rahimi, K.; Wessling, M. Phys. Chem. Chem. Phys. 2015, 17, 3768. (b) Yang, D.; Chen, T.; Wang, Z. J. Mater. Chem. A 2017, 5, 18967. (c) Zhao, Z. M.; Luo, S.; Ma, P.; Luo, Y. T.; Wu, W.; Long, Y.; Ma, J. T. ACS Sustain. Chem. Eng. 2020, 8, 8814.

[17] Gibert, R.; Rioux, R.; Saheb, E. Anal. Chem. 1984, 56, 106.

[18] (a) Lazouski, N.; Chung, M. J.; Williams, K.; Gala, M. L.; Manthiram, K. Nat. Catal. 2020, 3, 463. (b) Liu, D. N.; Wang, J. H.; Bian, S.; Liu, Q.; Gao, Y. H.; Wang, X.; Chu, P. K.; Yu, X. F. Adv. Funct. Mater. 2020, 2002731.

[19] Zhou, L.; Boyd, C. E. Aquaculture 2016, 450, 187

[20] Cui, X. Y.; Tang, C.; Zhang, Q. Adv. Energy Mater. 2018, 1800369.

[21] Hodgetts, R. Y.; Kiryutin, A. S.; Nichols, P.; Du, H. L.; Bakker, J. M.; Macfarlane, D. R.; Simonov, A. N. ACS Energy Lett. 2020, 5, 736.

[22] Suryanto, B. H. R.; Du, H. L.; Wang, D. B.; Chen, J.; Simonov, A. N.; MacFarlane, D. R. Nat. Catal. 2019, 2, 290.

[23] Shi, M. M.; Bao, D.; Wulan, B. R.; Li, Y. H.; Zhang, Y. F.; Yan, J. M.; Jiang, Q. Adv. Mater. 2017, 29, 1606550.

[24] Schlesinger, W. H.; Hartley, A. E. Biogeocemistry 1992, 15, 191.

[25] Turner, C.; Spanel, P.; Smith, D. A. Physiol. Meas. 2006, 27, 321

[26] Andersen, S. Z.; Colic, V.; Yang, S.; Schwalbe, J. A.; Nielander, A. C.; McEnaney, J. M.; Enemark-Rasmussen, K.; Baker, J. G.; Singh, A. R.; Rohr, B. A.; Statt, M. J.; Blair, S. J.; Mezzavilla, S.; Kibsgaard, J.; Vesborg, P. C. K.; Cargnello, M.; Bent, S. F.; Jaramillo, T. F.; Stephens, I. E. L.; Norskov, J. K.; Chorkendorff, I. Nature 2019, 570, 504

[27] (a) Aika, K.; Hori, H.; Ozaki, A. J. Catal. 1972, 27, 424. (b) Gong, Y. T.; Wu, J. Z.; Kitano, M.; Wang, J. J.; Ye, T. N.; Li, J.; Kobayashi, Y.; Kishida, K.; Abe, H.; Niwa, Y.; Yang, H. S.; Tada, T.; Hosono, H. Nat. Catal. 2018, 1, 178.

[28] Imbihl, R.; Behm, R. J.; Etrl, G.; Moritz, W. Surf. Sci. 1982, 123, 129.

[29] Somorjai, G.; Materer, N. Top. Catal. 1994, 1, 215.

[30] Zheng, J. W.; Liao, F. L.; Wu, S.; Jones, G.; Chen, T. Y.; Fellowes, J.; Sudmeier, T.; McPherson, I. J.; Wilkinson, I.; Tsang, S. C. E. Angew. Chem. Int. Ed. 2019, 58, 17335.

[31] Chen, S. M.; Perathoner, S.; Ampelli, C.; Mebrahtu, C.; Su, D. S.; Centi, G. Angew. Chem. Int. Ed. 2017, 56, 2699.

[32] Qiao, B. T.; Wang, A. Q.; Yang, X. F.; Allard, L. F.; Jiang, Z.; Cui, Y. T.; Liu, J. Y.; Li, J.; Zhang, T. Nat. Chem. 2011, 3, 634.

[33] Zhang, S. B.; Jin, M.; Shi, T. F.; Han, M. M.; Sun, Q.; Lin, Y.; Ding, Z. H.; Zheng, L. R.; Wang, G. Z.; Zhang, Y. X.; Zhang, H. M.; Zhao, H. J. Angew. Chem. Int. Ed. 2020, 59, 13423.

[34] Wang, M. F.; Liu, S. S.; Qian, T.; Liu, J.; Zhou, J. Q.; Ji, H. Q.; Xiong, J.; Zhong, J.; Yan, C. L. Nat. Commun. 2019, 10, 341.

[35] Kitano, M.; Inoue, Y.; Yamazaki, Y.; Hayashi, F.; Kanbara, S.; Matsuishi, S.; Yokoyama, T.; Kim, S. W.; Hara, M.; Hosono, H. Nat. Chem. 2012, 4, 934.

[36] Dahl, S.; Logadottir, A.; Egeberg, R. C.; Larsen, J. H.; Chorkendorff, I.; Tornqvist, E.; Norskov, J. K. Phys. Rev. Lett. 1999, 83, 1814.

[37] (a) Szmigiel, D.; Rarog-Pilecka, W.; Miskiewicz, E.; Glinski, M.; Kielak, M.; Kaszkur, M.; Kowalczyk, Z. Appl. Catal. A-Gen. 2004, 273, 105. (b) Rarog-Pilecka, W.; Miskiewicz, E.; Szmigiel D.; Kowalczyk, Z. J. Catal. 2005, 231, 11 .

[38] Peng, W.; Luo, M.; Xu, X.D.; Jiang, K.; Peng, M.; Chen, D. C.; 
Chan, T. S.; Tan, Y. W. Adv. Energy Mater. 2020, 2001364.

[39] Geng, Z. G.; Liu, Y.; Kong, X. D.; Li, P.; Li, K.; Liu, Z. Y.; Du, J. J.; Shu, M.; Si, R.; Zeng, J. Adv. Mater. 2018, 30, 1803498.

[40] Gronberg, K. L. C.; Gormal, C. A.; Durrant, M. C.; Smith, B. E.; Henderson, R. A. J. Am. Chem. Soc. 1998, 120, 10613.

[41] Sippel, D.; Rohde, M.; Netzer, J.; Trncik, C.; Gies, J.; Grunau, K.; Djurdjevic, I.; Decamps, L.; Andrade, S. L. A.; Einsle, O. Science 2018, 359, 1484 .

[42] Zhao, J. X.; Chen, Z. F. J. Am. Chem. Soc. 2017, 139, 12480.

[43] Han, L. L.; Liu, X. J.; Chen, J. P.; Lin, R. Q.; Liu, H. X.; Lu, F.; Bak, S.; Liang, Z. X.; Zhao, S. Z.; Stavitski, E.; Luo, J.; Adzic, R. R.; Xin, H. L. Angew. Chem. Int. Ed. 2019, 58, 2321.

[44] Huang, Y.; Yang, T. T.; Yang, L.; Liu, R.; Zhang, G. Z.; Jiang, J.; Luo, Y.; Lian, P.; Tang, S. B. J. Mater. Chem. A 2019, 7, 15173.

[45] Feng, J.; Zhu, X. J.; Chen, Q. Y.; Xiong, W.; Cheng, X.; Luo, Y. L.; Alshehri, A. A.; Alzahrani, K. A.; Jiang, Z. J.; Li, W. J. Mater. Chem. A 2019, 7, 26227.

[46] Zhang, X. P.; Kong, R. M.; Du, H. T.; Xia, L.; Qu, F. L. Chem. Commun. 2018, 54, 5323.

[47] Du, H. L.; Gengenbach, T. R.; Hodgetts, R.; MacFarlane, D. R.; Simonov, A. N. ACS Sustainable Chem. Eng. 2019, 7, 6839.

[48] Shi, M. M.; Bao, D.; Li, S. J.; Wulan, B. R.; Yan, J. M.; Jiang, Q. Adv. Energy Mater. 2018, 8, 1800124

[49] (a) Haruta, M.; Kobayashi, T.; Sano, H.; Yamada, N. Chem. Lett. 1987, 16, 405. (b) Ciriminna, R.; Falletta, E.; Della Pina, C.; Teles, J. H. Angew. Chem. Int. Ed. 2016, 55, 14210.

[50] Bao, D.; Zhang, Q.; Meng, F. L.; Zhong, H. X.; Shi, M. M.; Zhang, Y.; Yan, J. M.; Jiang, Q.; Zhang, X. B. Adv. Mater. 2017, 29, 1604799.

[51] Wang, X. Q.; Wang, W. Y.; Qiao, M.; Wu, G.; Chen, W. X.; Yuan, T. W.; Xu, Q.; Chen, M.; Zhang, Y.; Wang, X. L.; Wang, J.; Ge, J. J.; Hong, X.; Li, Y. F.; Wu, Y.; Li, Y. D. Sci. Bull. 2018, 63, 1246.

[52] (a) Dehcheshmeh, M. M.; Shervedani, R. K.; Torabi, M. Electrochim. Acta 2019, 327, 134895. (b) Li, D.; Chen, X. F.; Lv, Y. Z.; Zhang, G. Y.; Huang, Y.; Liu, W.; Li, Y.; Chen, R. S.; Nuckolls, C.; Ni, H. W. Appl. Catal. B-Environ. 2020, 269, 118824. (c) Luo, Y. P.; Huang, D. K.; Li, M.; Xiao, X.; Shi, W. N.; Wang, M. K.; Su, J.; Shen, Y. Electrochim. Acta 2016, $219,187$.

[53] Hao, R.; Sun, W. M.; Liu, Q.; Liu, X. L.; Chen, J. L.; Lv, X. W.; Li, W.; Liu, Y. P.; Shen, Z. R. Small 2020, 16, 2000015.

[54] (a) Wu, T. W.; Zhao, H. T.; Zhu, X. J.; Xing, Z.; Liu, Q.; Liu, T.; Gao, S. Y.; Liu, S. Y.; Chen, G.; Asiri, A. M.; Zhang, Y. N.; Sun, X. P. Adv. Mater. 2020, 2000299. (b) Lv, X.; Wang, F. Y.; Du, J.; Liu, Q.; Luo, Y. S.; Lu, S. Y.; Chen, G.; Gao, S. Y.; Zheng, B. Z. Sun, X. P. Sustain. Energy Fuels 2020, 4, 4469. (c) Xia, L.; Li, B. H.; Zhang, Y.; Zhang, R.; Ji, L.; Chen, H. Y.; Cui, G. W.; Zheng, H. G.; Sun, X. P.; Xie, F. Y.; Liu, Q. Inorg. Chem. 2019, 58, 2257. (d) Li, C. B.; Yu, J. L.; Yang, L.; Zhao, J. X.; Kong W. H.; Wang, T.; Asiri, A. M.; Li, Q.; Sun, X. P. Inorg. Chem. 2019, 58, 15, 9597. (e) Li, C. B.; Ma, D. W.; Mou, S. Y.; Luo, Y. S.; Ma, B. Y.; Lu, S. Y.; Cui, G. W.; Li, Q.; Liu, Q.; Sun, X. P. J. Energy Chem. 2020, 50, 402.

[55] Li, L. Q.; Tang, C.; Xia, B. Q.; Jin, H. Y.; Zheng, Y.; Qiao, S. Z. ACS Catal. 2019, 9, 2902.

[56] Zhang, R.; Ji, L.; Kong, W. H.; Wang, H. B.; Zhao, R. B.; Chen, H. Y.; Li, T. S.; Li, B. H.; Luo, Y. L.; Sun, X. P. Chem. Commun. 2019, $55,5263$.

[57] Han, Z.; Choi, C.; Hong, S.; Wu, T.; Soo, Y.; Yung, Y.; Qiu, J.; Sun, Z. Appl. Catal. B-Environ. 2019, 257, 117896.

[58] (a) Zhang, Y. Q.; Ouyang, B.; Xu, J.; Chen, S.; Rawat, R. S.; Fan, H. J. Adv. Energy Mater. 2016, 6, 1600221. (b) Hammer, B.; Norskov, J. K. Nature 1995, 376, 238.

[59] Zhang, L.; Ji, X. Q.; Ren, X.; Ma, Y. J.; Shi, X. F.; Tian, Z. Q.; Asiri, A. M.; Chen, L.; Tang, B.; Sun. X. P. Adv. Mater. 2018, 30, 1800191.

[60] Ren, X.; Cui, G. W.; Chen, L.; Xie, F. Y.; Wei, Q.; Tian, Z. Q.; Sun, X. P. Chem. Commun. 2018, 54, 8474.

[61] Zhang, R.; Guo, H. R.; Yang, L.; Wang, Y.; Niu, Z. G.; Huang, H.;
Chen, H. Y.; Xia, L.; Li, T. S.; Shi, X. F.; Sun, X. P.; Li, B. H.; Liu, Q. ChemElectroChem 2019, 6, 1014.

[62] Cheng, X.; Wang, J. W.; Xiong, W.; Wang, T.; Wu, T. W.; Lu, S. Y.; Chen, G.; Gao, S. Y.; Shi, X. F.; Jiang, Z. J.; Niu, X. B.; Sun, X. P. ChemNanoMat 2020, 6, 1315 .

[63] (a) Wang, Y. H.; Cui, X. Q.; Zhang, Y. Y.; Zhang, L. J.; Gong, X. G.; Zheng, G. F. Adv. Mater. 2016, 28, 7626. (b) Chen, G. F.; Cao, X. R.; Wu, S. Q.; Zeng, X. Y.; Ding, L. X.; Zhu, M.; Wang, H. H. J. Am. Chem. Soc. 2017, 139, 9771.

[64] Lee, H. K.; Koh, C. S. L.; Lee, Y. H; Liu, C.; Phang, I. Y.; Han, X. M.; Tsung, C. K.; Ling, X. Y. Sci. Adv. 2018, 4, 3208.

[65] Tong, Y. Y.; Guo, H. P.; Liu, D. L.; Yan, X.; Su, P. P.; Liang, J.; Zhou, S.; Liu, J.; Lu, G. Q.; Dou, S. X. Angew. Chem. Int. Ed. 2020, $132,7356$.

[66] Tao, H. C.; Choi, C.; Ding, L. X.; Jiang, Z.; Hang, Z. S.; Jia, M. W.; Fan, Q.; Gao, Y. N.; Wang, H. H.; Robertson, A. W.; Hong, S.; Jung, Y. S.; Liu, S. Z.; Sun, Z. Y. Chem 2019, 5, 204.

[67] Vojvodic, A.; Medford, A. J.; Studt, F.; Abild-Pedersen, F.; Khan, T. S.; Bligaard, T.; Norskov, J. K. Chem. Phys. Lett. 2014, 598, 108.

[68] (a) Nazemi, M.; El-Sayed, M. A. J. Phys. Chem. C 2019, 123, 11422. (b) Manjuatha, R.; Schechter, A. Electrochem. Commun. 2018, 90, 96. (c) Chang, B.; Deng, L. Q.; Wang, S. Z.; Shi, D.; Ai, Z. Z.; Jiang, H. H.; Shao, Y. L.; Zhang, L.; Shen, J. X.; Wu, Y. Z.; Hao, X. P. J. Mater. Chem. A 2020, 8, 91 .

[69] Qin, B. H.; Li, Y. H.; Zhang, Q.; Yang, G. X.; Liang, H.; Peng, F. Nano Energy 2020, 68, 104374.

[70] (a) Kowalczyk, Z.; Sentek, J.; Jodzis, S.; Muhler, M.; Hinrichsen, O. J. Catal. 1997, 169, 407. (b) Kowalczyk, Z. Catal. Lett. 1996, 37, 173. (c) Narasimharao, K.; Seetharamulu, P.; Rao, K. S. R.; Basahel, S. N. J. Mol. Catal. A-Chem. 2016, 411, 157.

[71] Hao, Y. C.; Guo, Y.; Chen, L. W.; Shu, M.; Wang, X. Y.; Bu, T. A.; Gao, W. Y.; Zhang, N.; Su, X.; Feng, X.; Zhou, J. W.; Wang, B.; Hu, C. W.; Yin, A. X.; Si, R.; Zhang, Y. W.; Yan, C. H. Nat. Catal. 2019 2,448 .

[72] (a) Su, L.; Han, D. D.; Zhu, G. J.; Xu, H.; Luo, W.; Wang, L. J.; Jiang, W.; Dong, A. G.; Yang, J. P. Nano Lett. 2019, 19, 5423. (b) Guillette, L. J.; Edwards, T. M. Integr. Comp. Biol. 2005, 45, 19.

[73] (a) Dima, G. E.; Beltramo, G. L.; Koper, M. T. M. Electrochim. Acta 2005, 50, 4318. (b) DeVooy, A. C. A.; Koper, M. T. M.; Van Santen, R. A.; Van Veen J. A. R. J. Electroanal. Chem. 2001, 506, 127. (c) Ye, T.; Durkin, D. P.; Banek, N. A.; Wagner, M. J.; Shuai, D. M. ACS Appl. Mater. Interfaces 2017, 9, 27421. (d) Zhang, R.; Shuai, D.; Guy, K. A.; Shapley, J. R.; Strathmann, T. J.; Werth, C. J. ChemCatChem 2013, 5, 313.

[74] Zhang, X.; Wang, Y. T.; Liu, C. B.; Yu, Y. F.; Liu, S. Y.; Zhang, B. Chem. Eng. J. 2021, 403, 126269.

[75] Wang, Y. T.; Zhou, W.; Jia, R. R.; Yu, Y. F.; Zhang, B. Angew. Chem. Int. Ed. 2020, 59, 5350 .

[76] Li, J.; Zhan, G. M.; Yang, J. H.; Quan, F. J.; Mao, C. L.; Liu, Y.; Wang, B.; Lei, F. C.; Li, L. J.; Chan, A. W. M.; Xu, L. P.; Shi, Y. B.; Du, Y.; Hao, W. C.; Wong, P. K.; Wang, J. F.; Dou, S. X.; Zhang, L. Z.; Yu, J. C. J. Am. Chem. Soc. 2020, 142, 7036.

[77] Wang, Y. T.; Yu, Y. F.; Jia, R. R.; Zhang, C.; Zhang, B. Natl. Sci. Rev. 2019, 6, 730.

[78] (a) Zhu, L.; Zhang, L.; Qu, H. X,; Zhong, Q. J. Mol. Catal. A-Chem. 2015, 409, 207. (b) Fritz, A.; Pitchon, V. Appl. Catal. B-Environ. 1997, 13, 1 .

[79] (a) Koebel, M.; Elsener, M.; Madia, G. Ind. Eng. Chem. Res. 2001, 40, 52. (b) Koebel, M. J. Catal. 2002, 209, 159.

[80] Long, J.; Chen, S. M.; Zhang, Y. L.; Guo, C. X.; Fu, X. Y.; Deng, D. H.; Xiao, J. P. Angew. Chem. Int. Ed. 2020, 59, 9711.

[81] (a) Li, R. G.; Zhang, F. X.; Wang, D. G.; Yang, J. X.; Li, M. R.; Zhu, J.; Zhou, X.; Han, H. X.; Li, C. Nat. Commun. 2013, 4, 1432. (b) Dong, B. B.; Cui, J. Y.; Gao, Y. Y.; Qi, Y.; Zhang, F. X.; Li, C. Adv. Mater. 2019, 31, 1808185 . 